\title{
Quadric SFDI for Laplacian Discretisation in Lagrangian Meshless Methods
}

\author{
Shiqiang $\operatorname{Yan}^{1} \cdot$ Q. W. Ma ${ }^{1} \cdot$ Jinghua Wang ${ }^{1}$ \\ Received: 7 September 2019 / Accepted: 12 May 2020 / Published online: 14 October 2020 \\ (C) The Author(s) 2020
}

\begin{abstract}
In the Lagrangian meshless (particle) methods, such as the smoothed particle hydrodynamics (SPH), moving particle semi-implicit (MPS) method and meshless local Petrov-Galerkin method based on Rankine source solution (MLPG_R), the Laplacian discretisation is often required in order to solve the governing equations and/or estimate physical quantities (such as the viscous stresses). In some meshless applications, the Laplacians are also needed as stabilisation operators to enhance the pressure calculation. The particles in the Lagrangian methods move following the material velocity, yielding a disordered (random) particle distribution even though they may be distributed uniformly in the initial state. Different schemes have been developed for a direct estimation of second derivatives using finite difference, kernel integrations and weighted/moving least square method. Some of the schemes suffer from a poor convergent rate. Some have a better convergent rate but require inversions of high order matrices, yielding high computational costs. This paper presents a quadric semi-analytical finite-difference interpolation (QSFDI) scheme, which can achieve the same degree of the convergent rate as the best schemes available to date but requires the inversion of significant lower-order matrices, i.e. $3 \times 3$ for $3 \mathrm{D}$ cases, compared with $6 \times 6$ or $10 \times 10$ in the schemes with the best convergent rate. Systematic patch tests have been carried out for either estimating the Laplacian of given functions or solving Poisson's equations. The convergence, accuracy and robustness of the present schemes are compared with the existing schemes. It will show that the present scheme requires considerably less computational time to achieve the same accuracy as the best schemes available in literatures, particularly for estimating the Laplacian of given functions.
\end{abstract}

Keywords Laplacian discretisation · Lagrangian meshless methods · QSFDI · Random/disordered particle distribution · Poisson's equation $\cdot$ Patch tests

\section{Introduction}

\section{Article Highlights}

- It develops a new scheme, QSFDI, for numerical interpolation, gradient calculation and Laplacian estimations using disordered particles, which is more efficient than others at the same accuracy level.

- The QSFDI can achieve the same degree of the convergent rate as the best scheme but requires inversing significant lower-order matrices, i.e. $3 \times 3$ for $3 \mathrm{D}$ cases, compared with $6 \times 6$ or $10 \times 10$ in the latter, and, consequently, requires much less CPU time.

- It presents a systematic patch test comparing different schemes in terms of accuracy, convergence and robustness.

Q. W. Ma

q.ma@city.ac.uk

1 School of Mathematics, Computer Science and Engineering, University of London, EC1V 0HB, London, UK
In the Lagrangian meshless/particle methods, for example, the smoothed particle hydrodynamics (SPH, e.g. Monaghan 1994; Shao and Lo 2003; Shao et al. 2006; Khayyer et al. 2008; Lind et al. 2012), moving particle semi-implicit method (MPS, e.g. Koshizuka and Oka 1996; Gotoh and Khayyer 2016; Khayyer and Gotoh 2010), the meshless local PetrovGalerkin method (MLPG, e.g., Ma 2005a, b; Zhou and Ma 2010), interpolating element-free Galerkin method (Abbaszadeh and Dehghan 2019; Dehghan and Abbaszadeh $2018,2019)$, the computational domain is represented by particles and the governing equations with associated boundary conditions are discretised to form a linear algebraic equation system, which leads to the approximation of physical quantities at particle locations. These methods become more 
popular, attributing to their superiority over the conventional mesh-based methods, such as the finite element method and finite volume method, in dealing with various engineering problems with large deformations, such as the breaking wave impact on offshore structures, for which the mesh-based methods may suffer from significant mesh distortions and/or numerical diffusions.

One key technique for the Lagrangian meshless methods is to numerically formulate and discretise the Laplacian operator, which is required mainly for three purposes. The first one is to discretise the governing equations, involving secondorder partial differential terms or Laplacian operators, e.g. the partial differential equation for the heat conduction and thermal diffusion (Chen et al. 1999; Schwaiger 2008), the pressure Poisson's equation employed by the projectionbased/fractional step methods for solving the Navier-Stokes (NS) equations to deal with fluid-structure interaction problems (Shao and Lo 2003; Ma et al. 2016) and the Helmholtz equation widely used in wave and diffusion problems. The second one is to numerically estimate specific physical quantities, which are expressed by the Laplacian of others, e.g., the viscous stress in the NS equation (Zheng et al. 2018). The third one is to discretise the Laplacian utilised as stabilisation operators to enhance the pressure calculation in various applications (Khayyer and Gotoh 2010; Khayyer and Gotoh 2012; Ikari et al. 2015). In meshless methods, the Laplacian of a function at a specific location is numerically formulated in terms of discretised function values at surrounding particles within the support domain using finite difference, kernel integration, moving least squares (MLS) or weighted least squares (WLS) algorithms. Therefore, the accuracy, convergence and computational efficiency of the schemes are significantly influenced by the particle distribution. In Eulerian meshless methods for steady problems (e.g., Lind and Stansby 2016), the particles are fixed and may be placed uniformly and regularly. For such problems, high-order finite difference schemes based on a uniform particle distribution can be directly applied to formulate the Laplacian operator. However, in the Lagrangian meshless methods for unsteady problems, the particles move at the material velocity and, consequently, their distribution may be highly disordered even though they may be placed regularly and uniformly in the initial state. Such disorderliness/randomness of the particle distribution considerably downgrades the schemes based on a uniform particle distribution.

For a random/disordered particle distribution, efforts have been devoted to the reviews of various schemes, e.g. on dealing with the viscous term in SPH (Zheng et al. 2018) and on solving pressure Poisson's equation (PPE) involved in projection-based meshless methods (Ma et al. 2016). It has been observed that some schemes (classified as type 1 by Ma et al. 2016), e.g. Cummins and Rudman (1999); Lo and Shao (2002); Lee et al. (2008); Xu et al. (2009); Hu and
Adams (2007); Gotoh et al. (2014); and Khayyer and Gotoh (2010, 2012), converge at a rate less than first order for estimating the Laplacian of a given function, although they do not need inversions of any matrices and thus have relatively low computational costs. Their performances may be improved by reducing the randomness of the particle distribution, e.g., using the particle shifting scheme proposed by Lind et al. (2012), or by introducing error correction and compensating terms, e.g. Oger et al. (2007) and Ikari et al. (2015), which requires the inversion of matrices. Alternatively, high-order Laplacian discretisation schemes have also been developed for a random/distorted particle distribution. These include the CSPM proposed by Chen et al. (1999), a scheme proposed by Fatehi and Manzari (2011), which was developed from the Brookshaw's scheme (Brookshaw 1985) by introducing an error compensation, the LSMPS developed by Tamai and Koshizuka (2014) using the MLS algorithm, and LP-MPS proposed by Tamai et al. (2017) using the WLS algorithm. These schemes are classified as type 3 schemes by Ma et al. (2016). Patch tests by Schwaiger (2008), Zheng et al. (2014) and Tamai et al. (2017) have shown that the CSPM, LP-MPS and quadric LSMPS have a higher convergent rate, compared with the type 1 schemes. However, formulating these schemes requires a significant computational cost on inversing matrices at all particle locations and at every time step of the transient simulations. For each particle in 3D problems, the CSPM and LP-MPS need to inverse two matrices with sizes of $6 \times 6$ and 3 $\times 3$, respectively; the quadric LSMPS needs to inverse a matrix with a size of $10 \times 10$. To overcome this problem, Schwaiger (2008) proposed a CSPH scheme, which is based on the CSPM but reduces the sizes of inversed matrices to two $3 \times 3$ for 3 D problems, through ignoring the cross-derivative terms of the 2 nd derivatives (thus downgrading the accuracy).

In this paper, a quadric semi-analytical finite-difference interpolation scheme (referred to as QSFDI) for numerically formulating the Laplacian operator is developed based on the principle of the linear SFDI (Ma 2008). Its consistency, accuracy and convergence property are similar to the quadric LSMPS and LP-MPS, for randomly distributed particles, whereas the sizes of the matrices to be inversed are considerably reduced to $3 \times 3$ for $3 \mathrm{D}$ problems, compared with $6 \times 6$ in the quadric LSMPS and LP-MPS. The performance of the present scheme will be assessed by systematic patch tests considering both directly estimating the Laplacian of specific functions and solving Poisson's equation.

\section{Mathematical Formulation}

Ma (2008) and Ma et al. (2016) developed the linear SFDI, which was based on Taylor's expansion with a leading truncation term of 2 nd derivatives, for numerical interpolations and gradient estimations. The patch test by Ma (2008) 
suggested that the linear SFDI is superior over the linear MLS when the particles are randomly distributed. The principle of the SFDI is extended here to derive the interpolation, gradient estimation and Laplacian discretisation schemes with a quadric accuracy.

For each particle $j$ at $\boldsymbol{x}_{j}$, which locates inside the support domain of Point $\boldsymbol{x}_{I}$, a function $p$ can be expressed as Taylor's expansion,

$$
\begin{aligned}
p_{j}-p_{I} & =\left.\left(\boldsymbol{r}_{j I}^{\mathrm{T}} \nabla\right) p(\boldsymbol{x})\right|_{\boldsymbol{x}=\boldsymbol{x}_{I}}+\left.\frac{1}{2}\left(\boldsymbol{r}_{j I}^{(2 s)}\right)^{\mathrm{T}} \nabla^{(2 s)} p(\boldsymbol{x})\right|_{\boldsymbol{x}=\boldsymbol{x}_{I}} \\
& +\left.\left(\boldsymbol{r}_{j I}^{(2 c)}\right)^{\mathrm{T}} \nabla^{(2 \boldsymbol{c})} p(\boldsymbol{x})\right|_{\boldsymbol{x}=\boldsymbol{x}_{I}}+\left.\frac{1}{6}\left(\boldsymbol{r}_{j I}^{\mathrm{T}} \nabla\right)^{3} p(\boldsymbol{x})\right|_{\boldsymbol{x}=\boldsymbol{x}_{I}}+\ldots
\end{aligned}
$$

where $\boldsymbol{x}=\left[\begin{array}{lll}x & y & z\end{array}\right]^{\mathrm{T}}, \boldsymbol{r}_{j I}=\boldsymbol{x}_{j}-\boldsymbol{x}_{I}=\left[\begin{array}{lll}x_{j I} & y_{j I} & z_{j I}\end{array}\right]^{\mathrm{T}}$ and $\boldsymbol{\nabla}$ is the spatial differential operator. In Eq. (1), the 2nd derivative term $\frac{1}{2}\left(\boldsymbol{r}_{j I}^{T} \nabla\right){ }^{2} p(\boldsymbol{x})$ in the conventional Taylor's expansion (e.g. Chen et al. 1999; Tamai et al. 2017) is split into two, i.e. $\frac{1}{2}\left(\boldsymbol{r}_{j I}^{(2 s)}\right)^{\mathrm{T}} \boldsymbol{\nabla}^{(2 s)} p(\boldsymbol{x}) \quad$ and $\quad\left(\boldsymbol{r}_{j I}^{(2 c)}\right)^{\mathrm{T}} \nabla^{(2 c)} p(\boldsymbol{x}) \quad$ where $\boldsymbol{r}_{j I}^{(2 s)}=\left[\begin{array}{lll}x_{j I}^{2} & y_{j I}^{2} & z_{j I}^{2}\end{array}\right]^{\mathrm{T}}, \quad \boldsymbol{r}_{j I}^{(2 c)}=\left[\begin{array}{llll}x_{j I} & y_{j I} x_{j I} & z_{j I} y_{j I} & z_{j I}\end{array}\right]^{\mathrm{T}}$, $\nabla^{(2 s)}=\left[\begin{array}{lll}\frac{\partial^{2}}{\partial x^{2}} & \frac{\partial^{2}}{\partial y^{2}} & \frac{\partial^{2}}{\partial z^{2}}\end{array}\right]^{\mathrm{T}}, \quad$ a n d $\quad \nabla^{(2 c)}=\left[\begin{array}{lll}\frac{\partial^{2}}{\partial x \partial y} & \frac{\partial^{2}}{\partial x \partial z} & \frac{\partial^{2}}{\partial y \partial z}\end{array}\right]^{\mathrm{T}}$. Following Ma (2008), the weighted summation of Eq. (1) for all particles in the support domain of Point $\boldsymbol{x}_{I}$ are used to derive the schemes for approximating the gradient $\nabla p(\boldsymbol{x})$, 2nd derivatives and Laplacian $\nabla^{2} p(\boldsymbol{x})$, as well as the interpolation function for approximating $p(\boldsymbol{x})$ at $\boldsymbol{x}_{I}$. The details of the derivation are presented in Appendix 1. Only the final formulas are summarised below:

$$
\begin{array}{r}
\left\langle\left.\nabla p(\boldsymbol{x})\right|_{\boldsymbol{x}=\boldsymbol{x}_{I}}\right\rangle=\boldsymbol{M}_{1 q, I}^{-1} \sum_{j=1}^{N} \frac{w_{j I}}{d_{j I}^{2}} \boldsymbol{q}_{j I}\left(p_{j}-p_{I}\right)-\boldsymbol{M}_{1 q, I}^{-1} \sum_{j=1}^{N} \frac{w_{j I}}{d_{j I}^{2}} \boldsymbol{q}_{j I}\left(\boldsymbol{r}_{j I}^{(2 c)}\right)^{\mathrm{T}} \boldsymbol{M}_{2 c, I}^{-1} \\
\sum_{k=1}^{N} \frac{w_{k I}}{d_{k I}^{4}} \boldsymbol{r}_{k I}^{(2 c)}\left(p_{k}-p_{I}\right)-\boldsymbol{M}_{1 q, I}^{-1} \sum_{j=1}^{N} \frac{w_{j I}}{d_{j I}^{2}} \boldsymbol{q}_{j I} \boldsymbol{\Pi}_{j I}^{\mathrm{T}} \boldsymbol{M}_{2 s, I}^{-1} \sum_{k=1}^{N} \boldsymbol{\Gamma}_{k I}\left(p_{k}-p_{I}\right)
\end{array}
$$

$\left\langle\left.\nabla^{2} p(\boldsymbol{x})\right|_{\boldsymbol{x}=\boldsymbol{x}_{I}}\right\rangle=\boldsymbol{I}^{\mathrm{T}}\left\{2 \boldsymbol{M}_{2 s, I}^{-1} \sum_{j=1}^{N} \boldsymbol{\Gamma}_{j I}\left(p_{j}-p_{I}\right)-2 \boldsymbol{M}_{2 s, I}^{-1} \sum_{j=1}^{N} \frac{w_{j I}}{d_{j I}^{4}} \boldsymbol{\Pi}_{j l} \boldsymbol{G}_{j I}^{\mathrm{T}}\left\langle\left.\nabla p(\boldsymbol{x})\right|_{x=x_{I}}\right\rangle\right\}$

where \langle\rangle indicates an approximated value; $\boldsymbol{I}=\left[\begin{array}{lll}1 & 1 & 1\end{array}\right]^{\mathrm{T}}$; $w_{j I}$ is the weighting function for particle $j$ related to $\boldsymbol{x}_{I} ; d_{j I}$ is the distance between particle $j$ and $\boldsymbol{x}_{I}$; definitions of matrices $\boldsymbol{M}_{2 c, I}, \boldsymbol{M}_{2 s, I}, \boldsymbol{M}_{1 q, I}, \boldsymbol{q}_{j I}, \boldsymbol{\Gamma}_{j I}, \boldsymbol{\Pi}_{j I}$ and $\boldsymbol{G}_{j I}$ can be found in Appendix 1. In practices, Eq. (3) can be applied to discretising the Poisson's equation at all particle positions and/or to directly estimating $\nabla^{2} p(\boldsymbol{x})$ at point $\boldsymbol{x}_{I}$ coinciding with a particle location. However, if $\boldsymbol{x}_{I}$ does not coincide with any particles, the value $p_{I}$ needs to be numerically interpolated in order to estimate $\left.\nabla^{\mathbf{2}} p(\boldsymbol{x})\right|_{\boldsymbol{x}=\boldsymbol{x}_{I}}$. For this purpose, the interpolation function in the QSFDI is also derived and formulated in Appendix 1. Replacing $p_{I}$ in Eq. (3) by its approximated value $\left\langle p_{I}\right\rangle$ using Eq. (33), it leads to

$$
\left\langle\left.\nabla^{2} p(\boldsymbol{x})\right|_{x=\boldsymbol{x}_{I}}\right\rangle=\boldsymbol{I}^{\mathrm{T}}\left\{2 \boldsymbol{M}_{2 s, I}^{-1} \sum_{j=1}^{N} \boldsymbol{\Gamma}_{j I}\left(p_{j}-\left\langle p_{I}\right\rangle\right)-2 \boldsymbol{M}_{2 s, I}^{-1} \sum_{j=1}^{N} \frac{w_{j I}}{d_{j I}^{4}} \boldsymbol{\Pi}_{j I} \boldsymbol{G}_{j I}^{\mathrm{T}}\left\langle\left.\nabla p(\boldsymbol{x})\right|_{x=x_{I}}\right\rangle\right\}
$$

for approximating the Laplacian at a position that does not coincide with particles. The leading truncation errors for Eqs. (2) and (3) are

$\boldsymbol{E}_{\left.\nabla p(\boldsymbol{x})\right|_{x=x_{I}}}=-\left.\frac{1}{6} \boldsymbol{M}_{1 q, I}^{-1} \sum_{j=1}^{N} \frac{w_{j I}}{d_{j I}^{2}} \boldsymbol{q}_{j I}\left(\boldsymbol{F}_{j I}^{\mathrm{T}}-\boldsymbol{\Pi}_{j I}^{\mathrm{T}} \boldsymbol{M}_{2 s, I}^{-1} \sum_{k=1}^{N} \frac{w_{k I}}{d_{k I}^{4}} \boldsymbol{\Pi}_{k I} \boldsymbol{F}_{k I}^{\mathrm{T}}\right) \nabla^{(\mathbf{3})} p(\boldsymbol{x})\right|_{\boldsymbol{x}=\boldsymbol{x}_{I}}$

and

$\boldsymbol{E}_{\left.\nabla^{2} p(x)\right|_{x=x_{I}}}=\boldsymbol{I}^{\mathrm{T}}\left\{-\left.\frac{1}{3} \boldsymbol{M}_{2 s, I}^{-1} \sum_{j=1}^{N} \frac{w_{j I}}{d_{j I}^{4}} \boldsymbol{\Pi}_{j l} \boldsymbol{F}_{j I}^{\mathrm{T}} \boldsymbol{I}^{(3)} p(\boldsymbol{x})\right|_{\boldsymbol{x}=\boldsymbol{x}_{l}}-2 \boldsymbol{M}_{2 s, I}^{-1} \sum_{j=1}^{N} \frac{w_{j I}}{d_{j l}^{4}} \boldsymbol{\Pi}_{j l} \boldsymbol{G}_{j l}^{\mathrm{T}} \boldsymbol{E}_{\left.\nabla p(x)\right|_{x=x_{l}}}\right\}$

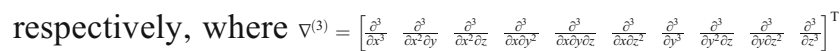
and the definition of matrix $\boldsymbol{F}_{j I}$ can be found in Appendix 1. For a random/disordered particle distribution, the leading truncation errors corresponding to the gradient estimation (Eq. (5)), the Laplacian discretisation (Eq. (6)) and the numerical interpolation to approximate $\left\langle p_{I}\right\rangle$ (Eq. (34)) are all proportional to $\left.\nabla^{(3)} p(\boldsymbol{x})\right|_{\boldsymbol{x}=\boldsymbol{x}_{I}}$. This implies that the QSFDI leads to the exact solution for quadric polynomials for the numerical interpolation, gradient estimation and Laplacian discretisation. The consistency of the QSFDI is the same as the LP-MPS developed by Tamai et al. (2017) and the quadric LSMPS (Quinlan et al. 2006). However, the error analysis on the CSPM (Chen et al. 1999) and Schwaiger's scheme (Schwaiger 2008) given in Appendix 2 suggests that their leading truncation errors is proportional to $\left.\nabla^{(2)} p(\boldsymbol{x})\right|_{\boldsymbol{x}=\boldsymbol{x}_{I}}$ for the cases with a random/disordered particle distribution. The consistency of these schemes is one-order lower than the QSFDI. It is worth noting that $\nabla^{(2)} p(\boldsymbol{x})$ in the leading truncation error of the CSPM, i.e. Eq. (42), origins from the leading truncation error of the gradient approximation scheme proposed in the CSPM, i.e. Eq. (40). This approximation can be replaced by a higher-order gradient estimation scheme, e.g. Eq. (48), to improve the accuracy and the consistency of the Laplacian discretisation, as demonstrated in Appendix 2. The formulation based on Eq. (48) is referred to as the ICSPM in the rest of the paper and 
summarised below,

$$
\begin{aligned}
& \left\langle\left.\nabla^{2} p(\boldsymbol{x})\right|_{\boldsymbol{x}=\boldsymbol{x}_{I}}\right\rangle=2 \boldsymbol{I}_{\mathrm{CSPM}}^{\mathrm{T}} \boldsymbol{M}_{2, \mathrm{ICSPM}}^{-1} \\
& \left\{\sum_{j=1}^{N} \frac{\nabla^{(2)} W_{j I} m_{j}}{\rho_{j}}\left(p_{j}-p_{I}\right)-\sum_{j=1}^{N} \frac{\nabla^{(2)} W_{j I} m_{j}}{\rho_{j}} \boldsymbol{r}_{j I}^{\mathrm{T}} \boldsymbol{M}_{1, \mathrm{CSPM}}^{-1} \sum_{k=1}^{N} \frac{\nabla W_{j I} m_{k}}{\rho_{k}}\left(p_{k}-p_{I}\right)\right\}
\end{aligned}
$$

where $m_{j} / \rho_{j}$ is the volume (area) represented by particle $j$; and $\boldsymbol{I}_{\mathrm{CSPM}}=\left[\begin{array}{llllll}1 & 0 & 0 & 1 & 0 & 1\end{array}\right]^{\mathrm{T}}$; the definitions of matrices $\boldsymbol{M}_{2, \text { ICSPM }}, \boldsymbol{M}_{2, \text { CSPM }}$ and $\boldsymbol{M}_{1, \text { CSPM }}$ are given in Appendix 2. The leading truncation error of Eq. (7) is

$\frac{1}{3} \boldsymbol{I}_{\mathrm{CSPM}}^{\mathrm{T}} \boldsymbol{M}_{2, \mathrm{CSPM}}^{-1}\left\{\left.\sum_{j=1}^{N} \frac{\nabla^{(2)} W_{j I} m_{j}}{\rho_{j}} \boldsymbol{r}_{j I}^{\mathrm{T}} \boldsymbol{M}_{1, \mathrm{CSPM}}^{-1} \sum_{k=1}^{N} \frac{\nabla W_{k I} m_{k}}{\rho_{k}}\left(\boldsymbol{r}_{k I}^{(3)}\right)^{\mathrm{T}} \nabla^{(3)} p(\boldsymbol{x})\right|_{\boldsymbol{x}=\boldsymbol{x}_{I}}-\left.\sum_{j=1}^{N} \frac{\nabla^{(2)} W_{j I} m_{j}}{\rho_{j}}\left(\boldsymbol{r}_{k I}^{(3)}\right)^{\mathrm{T}} \nabla^{(3)} p(\boldsymbol{x})\right|_{\boldsymbol{x}=\boldsymbol{x}_{I}}\right\}$

which is proportional to $\left.\nabla^{(3)} p(\boldsymbol{x})\right|_{\boldsymbol{x}=\boldsymbol{x}_{I}}$, the same as the quadric LSMPS, LP-MPS and the present QSFDI. It shall be noted that the leading truncation errors of the schemes discussed above are not only dominated by the terms of high-order derivatives (e.g. $\nabla^{(3)} p(x)$ for the QSFDI and ICSPM), which may reflect the accuracy level and the consistency, but also subjected to the corresponding orders of magnitudes, which indicates the convergence rates and are influenced by the mean particle spacing, $s_{0}$, the weighting/kernel function, the radius of the support domain and the randomness of the particle distributions. One may follow Quinlan et al. (2006) and Fatehi and Manzari (2011) to carry out a further theoretical analysis on the orders of magnitude of the truncation error; however, patch tests will be used in this paper to demonstrate the convergence properties of the QSFDI and the ICSMP.

Compared with the leading truncation error, it is equally important to look at the number and sizes of the matrices to be inversed in order to formulate the Laplacian operator. For the QSFDI, to approximate the gradient, the 2nd derivatives and the Laplacian of $p(\boldsymbol{x})$, three matrices, i.e. $\boldsymbol{M}_{2 c, I}, \boldsymbol{M}_{2 s, I}$ and $\boldsymbol{M}_{1 q}$, I, need to be inversed. For 3D problems, they all have sizes of $3 \times 3$, whereas for 2D problems, the sizes of $\boldsymbol{M}_{2 s, I}$ and $\boldsymbol{M}_{1 q, I}$ are $2 \times 2$ and $\boldsymbol{M}_{2 c, I}$ is a scalar. The sizes of matrices to be inversed for formulating the Laplacian in the QSFDI are slightly higher than the Schwaiger's scheme (e.g. CSPH2Г) for 3D problems but considerably lower than the LP-MPS, the quadric LSMPS and the CSPM, for which the number and the sizes of matrices to be inversed are summarised in Table 1. As mentioned above, an improved scheme for CSPM, i.e., ICSPM is also introduced. The number and sizes of the matrices to be inversed in the ICSPM are the same as the original CSPM, although the accuracy of the former is one order higher than the latter for a random particle distribution.

It is well known that the CPU time spent on the inversion of a matrix with size of $M \times M$ with $M$ being 2 to 10 is proportional to $M^{3}$. The CPU time spent on matrix inversions for $2 \mathrm{D}$ problems may be indicated by $\sim 2 \times 2^{3}$ for $\mathrm{CSPH} 2 \Gamma$ and QSFDI,
$2^{3}+3^{3}$ for CSPM, LP-MPS and ICSPM, $\sim 6^{3}$ for quadric LSMPS. The CPU time by the QSFDI on matrix inversions is approximately $45.7 \%$ of those by the LP-MPS or ICSPM, and $7.4 \%$ of that by the quadric LSMPS for $2 \mathrm{D}$ problems. The corresponding figures for 3D problems are $40 \%$ and $8.1 \%$, respectively. Nevertheless, different schemes require different CPU time on formulating the matrices; the overall robustness of these schemes will be investigated in the following patch tests.

\section{Patch Test}

To quantify the accuracy, convergence and robustness of the QSFDI and ICSPM, patch tests are performed using various cases. As indicated above, some of the main purposes for the Laplacian discretisation are (1) to find physical quantities, which may be expressed as the Laplacian of others, and (2) to discretise the Poisson's equations. For the former, the Laplacians of various specified functions $f(x, y)$, which are frequently used in literatures are directly estimated. For the latter, the Poisson's equation are considered:

$\nabla^{2} f(x, y)=($ R.H.S $)$

Table 1 Summary of the approaches for Laplacian discretisation

\begin{tabular}{lll}
\hline Schemes & \multicolumn{2}{l}{ Matrix inversion } \\
\cline { 2 - 3 } & 2 D problems & 3D problems \\
\hline CSPM (Eq. (42)) & One $3 \times 3$ and one $2 \times 2$ & One $6 \times 6$ and one $3 \times 3$ \\
CSPH2 $\Gamma$ (Eq. (48)) & Two $2 \times 2$ & Two $3 \times 3$ \\
Quadric LSMPS & One $6 \times 6$ & One $10 \times 10$ \\
LP-MPS & One $3 \times 3$ and one $2 \times 2$ & One $6 \times 6$ and one $3 \times 3$ \\
ICSPM (Eq. (7)) & One $3 \times 3$ and one $2 \times 2$ & One $6 \times 6$ and one $3 \times 3$ \\
QSFDI (Eq. (3)) & Two $2 \times 2$ & Three $3 \times 3$ \\
\hline
\end{tabular}


where the function denoted by $(R . H . S)$ is pre-specified as the real values of the Laplacian of $f(x, y)$. The present QSFDI, ICSPM and other existing schemes listed in Table 1 are employed for comparisons in the patch tests. When the ICSPM, CSPM and CSPH $2 \Gamma$ are performed, $m_{j} / \rho_{j}$ in their formulations is evaluated by using $1 / \sum_{j=1}^{N} W_{j I}$ ( $\mathrm{Hu}$ and Adams 2007). It is well known that the kernel/weighting functions play an important role in the convergence and accuracy of the numerical schemes in the meshless method (Quinlan et al. 2006), as demonstrated by the error analysis, e.g. Eqs. (5) and (6), which contains the weighting functions. The main aim of this paper is to introduce the QSFDI and ICSPM, and a quintic spline kernel function (Schwaiger 2008),

$$
W(r, h)=\frac{7}{478 \pi h^{2}}\left\{\begin{array}{cc}
\left(3-\frac{r}{h}\right)^{5}-6\left(2-\frac{r}{h}\right)^{5}+15\left(1-\frac{r}{h}\right)^{5}, & 0 \leq \frac{r}{h} \leq 1 \\
\left(3-\frac{r}{h}\right)^{5}-6\left(2-\frac{r}{h}\right)^{5}, & 1<\frac{r}{h} \leq 2 \\
\left(3-\frac{r}{h}\right)^{5}, & 2<\frac{r}{h} \leq 3 \\
0, & \frac{r}{h}>3
\end{array}\right.
$$

where $r=d_{j I}$ is used as the kernel function of ICSPM, CSPM and $\mathrm{CSPH} 2 \mathrm{\Gamma}$, and the weighting function of other schemes. By using Eq. (9), the radius of the support domain is $3 h$. The effect of the kernel/weighting functions will not be investigated and discussed in the future.

\subsection{Estimating Laplacian of Specified Functions}

Following Schwaiger (2008), the first group of the test functions used is

$p(x, y)=x^{m} y^{m}$

The computational domain is a unit square with $2 \leq x \leq 3$ and $2 \leq y \leq 3$. The particles are initially generated using a uniform spacing, i.e. $\Delta x=\Delta y=s_{0}$. To reflect the randomness of the particle distribution, a random shift with $\Delta x^{\prime}=K \delta s_{0}$ and $\Delta y^{\prime}=K \delta s_{0}$ is applied to all particles, where $K$ is a scale factor and $\delta$ is a random number between -1 and 1 . In the patch tests, $K$ is in the range of 0.1 to 0.8 . The Laplacian of the function $p$ at any particle $i,\left\langle\nabla^{2} p_{i}\right\rangle$, are estimated by different schemes summarised in Table 1 . The relative error is estimated in the same way as in Schwaiger (2008) for the sake of comparison, given by

$E_{r}=\sqrt{\sum_{i=1}^{N} \frac{m_{i}}{\rho_{i}}\left(\frac{\nabla^{2} p_{i}-\left\langle\nabla^{2} p_{i}\right\rangle}{\nabla^{2} p_{i}}\right)^{2}}$

where $\nabla^{2} p_{i}$ is the analytical value of the Laplacian of the function $p$ at Particle $i ; N$ is the total number of particles at which the Laplacian are estimated. It shall be pointed out that all existing schemes suffer from a downgraded accuracy when being applied to the particles near the boundaries due to the fact that the support domain is not full (Chen et al. 1999; Schwaiger 2008) or the neighbouring particles are mainly distributed in a quadrant or a half of the support domain of the particles. For simplicity, as well as being consistent with Schwaiger (2008), only the inner particles within a region of $2.25<x<2.75$ and $2.25<y<2.75$ are considered for the error evaluation using Eq. (11). Different $s_{0}$ (between 0.1 and 0.00025 ) and ratio $h / s_{0}$ (ranging from 0.75 to 1.2 ) are considered in the patch test. For specific $s_{0}, h / s_{0}$ and $K$, at least 10 repeated tests are carried out in order to eliminate the possible abnormality in the random series, the relative error and CPU times are taken as the mean values of the repeated tests.

Figure 1 compares the relative errors for estimating the Laplacian of $p(x, y)=x^{6} y^{6}$ using different schemes with a randomness specified by $K=0.4$. As can be seen, the LPMPS, quadric LSMPS and the present QSFDI result in a linear rate of reduction of the relative error as $s_{0}$ decreases, while the CSPM and CSPH2 $\Gamma$ do not seem to be convergent as $s_{0}$ decreases for a constant ratio of $h /$ $s_{0}$, conforming to the patch tests in Schwaiger (2008). Compared with CSPM, the relative error of the ICSPM not only is lower but also reduces linearly as $s_{0}$ decreases. It is also observed from Figure 1 that the present QSFDI leads to almost identical results as the LP-MPS, which are more accurate than other schemes, for all values of $h / s_{0}$ and $s_{0}$ applied in the patch test. As analysed in Section 2, the QSFDI demands less computational efforts on matrix inversions than the LP-MPS; the overall CPU time spent by the former is expected to be shorter than the latter. This is confirmed by Figure 2, which shows that the average CPU time spent by the QSFDI is approximately $20 \%$ less than that by the LP-MPS for achieving results with the same accuracy. In addition, Figure 3 displays the relative errors of the Laplacian discretisation against CPU times by different schemes in the cases with $K=0.4$ and different values of $h / s_{0}$. For convenience, the CPU times are scaled by the reference duration, $T_{\text {Ref }}$, which is the CPU time spent by the CSPH2 $\Gamma$ with $s_{0}=0.1$ and $h=$ $0.75 s_{0}$. Both Figures 2 and 3 confirm that the QSFDI requires considerably shorter CPU time than all other schemes for achieving a specific accuracy of estimating the Laplacian of function $p(x, y)=x^{6} y^{6}$.

It shall be pointed out that Schwaiger (2008) and Zheng et al. (2014) have adopted $h / s_{0}=0.268 / \sqrt{s_{0}}$ in the CSPM and CSPH2 $\Gamma$, based on the error analysis by Quinlan et al. (2006). A linear convergence was observed in their patch tests. By adopting such a strategy, the relative sizes of the support (kernel integration) domain 


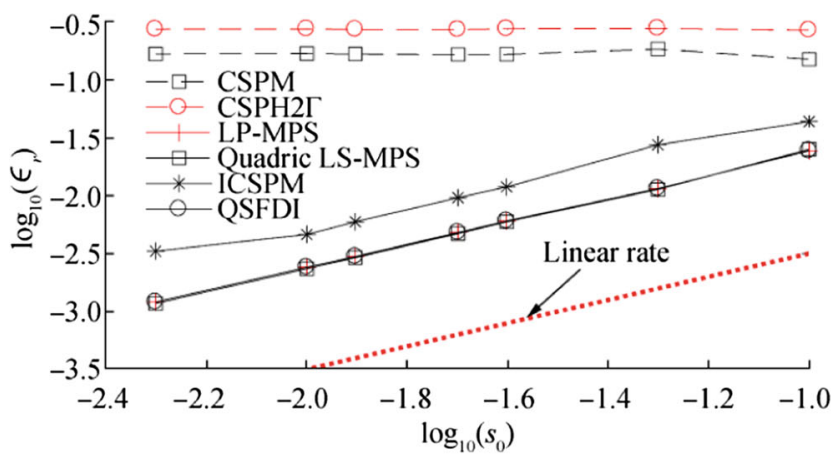

(a) $h=0.75 s_{0}$

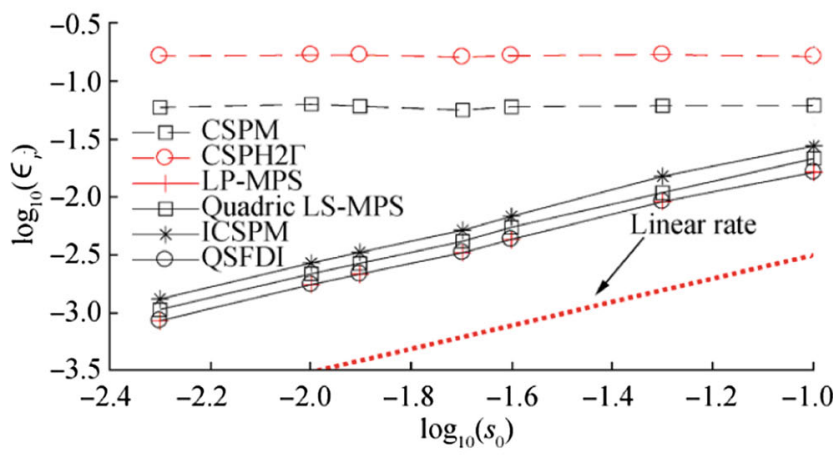

(b) $h=0.9 s_{0}$

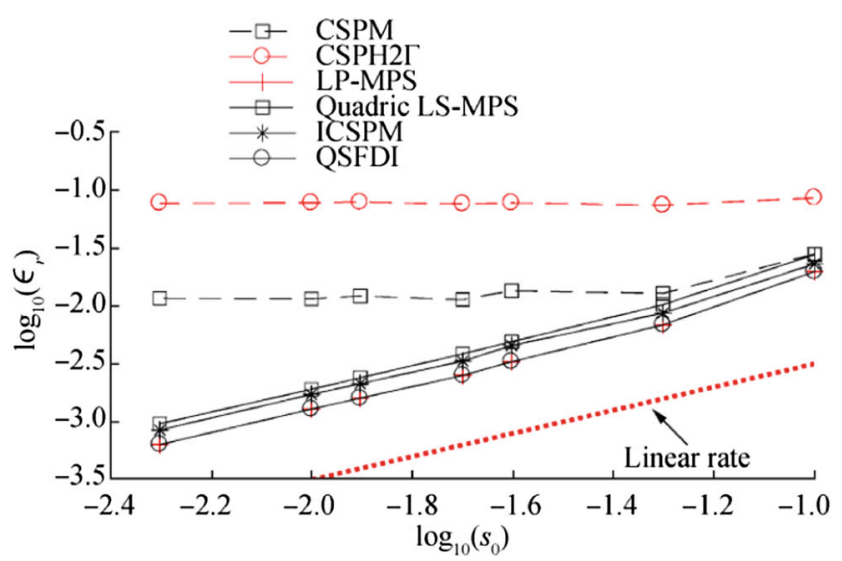

(c) $h=1.2 s_{0}$

Figure 1 Relative error of Laplacian discretisation $v s$ mean particle spacing $s_{0}$ for estimating Laplacian of $p(x, y)=x^{6} \mathrm{y}^{6}(K=0.4$; error estimation domain $2.25<x<2.75,2.25<y<2.75$ ). a $h=0.75 s_{0}$. b $h=0.9 s_{0} . \mathbf{c} h=1.2 s_{0}$

increase as $s_{0}$ decreases (e.g. $h / s_{0}$ are approximately 0.85 and 2.68 for $s_{0}=0.1$ and 0.01 , respectively). However, as the increase of $h / s_{0}$, more neighbouring particles are included in the kernel integration, yielding a considerably increase of CPU time spent on the Laplacian discretisation for each particle. In fact, not only the CSPM and $\mathrm{CSPH} 2 \mathrm{\Gamma}$, the accuracies and the robustness of all schemes may be significantly influenced by the ratio $h / s_{0}$. To further illustrate the effects of the ratio $h / s_{0}$ on the robustness

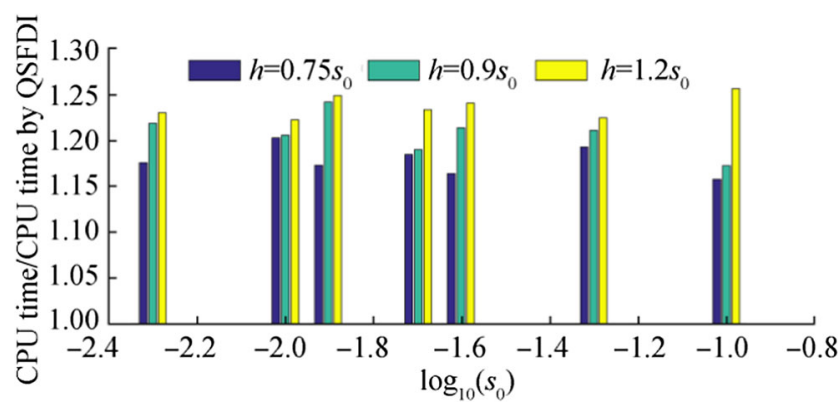

Figure 2 Ratio of the CPU time spent by the LP-MPS and that by the QSFDI on estimating Laplacian of $\boldsymbol{p}(\boldsymbol{x}, \boldsymbol{y})=\boldsymbol{x}^{\mathbf{6}} \boldsymbol{y}^{\mathbf{6}}(K=0.4)$

of the schemes, an alternative of Figure 3 is presented in Figure 4. For clarity, the corresponding results by the CSPH2 $\Gamma$, ICSPM and QSFDI are shown in Figure 4a; those by the CSPM, quadric LSMPS and the LP-MPS are shown in Figure $4 \mathrm{~b}$. It is observed that the robustness of the QSFDI, LP-MPS and quadric LSMPS is much less sensitive than that of CSPM, CSPH2 $\Gamma$, and ICSPM to the variation of $h / s_{0}$, though the robustness of all the schemes is improved with the increase of $h / s_{0}$. It implies that one may optimise the kernel/weighting function and the configuration of the $h / s_{0}$ to accelerate the convergent rates for these schemes. Nevertheless, it is not the focus of this paper and, therefore, only constant ratio $h / s_{0}$ is considered in the rest of the paper.

Similar behaviours to Figures 1 and 2 have been observed in the cases with other values of $K$. Some results are shown in Figures 5 and 6 for demonstration. To save the space, not all the results with different ratios of $h / s_{0}$ are presented. In Figure $5, h / s_{0}=1.2$ is used by the CSPM, CSPH2 $\Gamma$ and ICSPM; $h / s_{0}=0.75$ is adopted by others. In such a way, the CSPM, CSPH2 $\Gamma$, ICSPM and the LSMPS are expected to have the best robustness and the accuracy compared to other values of $h / s_{0}$, whereas the results of the QSFDI and the LP-MPS adopting $h / s_{0}=0.75$ are worse than the corresponding results with a greater $h / s_{0}$, as demonstrated by Figure 4 . Again, it is clearly seen that the QSFDI and the LPMPS lead to the highest accuracy and convergence properties compared to others. The comparison of the CPU time spent by the LP-MPS and the QSFDI in Figure 6 again confirms the superiority of the former in terms of saving CPU time for the cases with a different randomness.

Considering the fact that most of analytical formulations used in the engineering problems may be represented by hyperbolic/exponential and/or trigonometric functions (e.g. the velocity potential associated with a linear propagation wave and the temperature in 2D heat conduction problems), the exponential function applied by Tamai et al. (2017) 


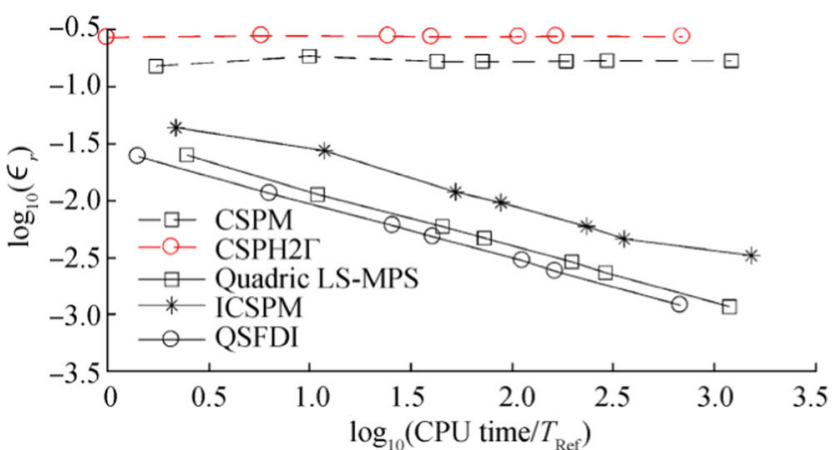

(a) $h=0.75 s_{0}$

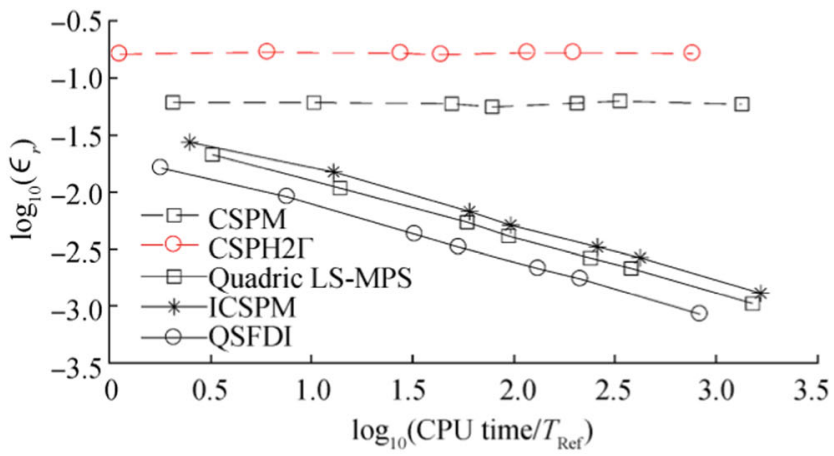

(b) $h=0.9 s_{0}$

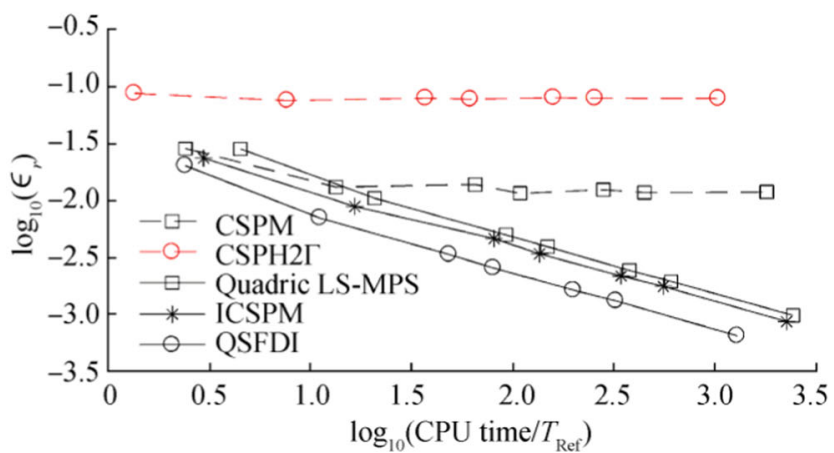

(c) $h=1.2 s_{0}$

Figure 3 Relative error of Laplacian discretisation $v_{s}$ CPU time for estimating Laplacian of $p(x, y)=x^{6} y^{6}\left(K=0.4 ; T_{\mathrm{Ref}}\right.$ is the CPU time spent by CSPH $2 \Gamma$ with $s_{0}=0.1$ and $h=0.75 s_{0}$; error estimation domain $2.25<x<2.75,2.25<y<2.75$ ). a $h=0.75 s_{0}$. b $h=0.9 s_{0}$. c $h=1.2 s_{0}$

$$
\begin{array}{r}
p(x, y)=\frac{3}{4} \exp \left(-\frac{(9 x-2)^{2}}{4}-\frac{(9 y-2)^{2}}{4}\right)+\frac{3}{4} \exp \left(-\frac{(9 x+1)^{2}}{49}-\frac{(9 y+1)^{2}}{10}\right) \\
+\exp \left(-\frac{(9 x-7)^{2}}{4}-\frac{(9 y-3)^{2}}{4}\right)-\frac{1}{5} \exp \left(-(9 x-4)^{2}-(9 y-7)^{2}\right)
\end{array}
$$

and a sine function employed by Zheng et al. (2014)

$p(x, y)=\sin (6 x+8 y)$

are also considered. In these tests, the computational domain is taken as a unit square with $0 \leq x \leq 1 ; 0 \leq y \leq 1$. The particle generation is the same as that used in the first test case, i.e. a

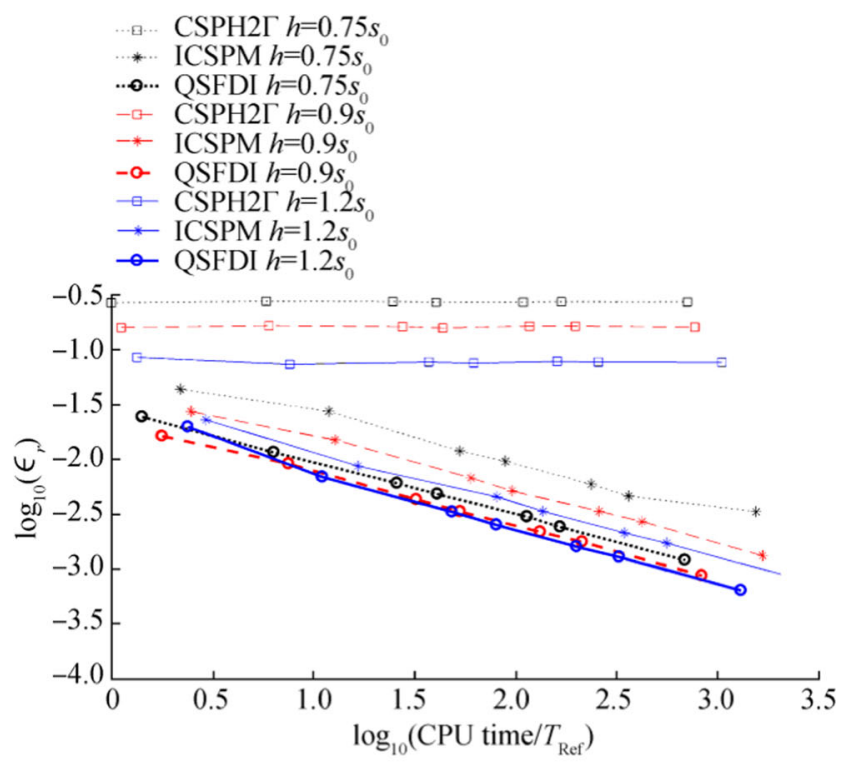

(a) CSPH2Г, ICSPM, QSFDI

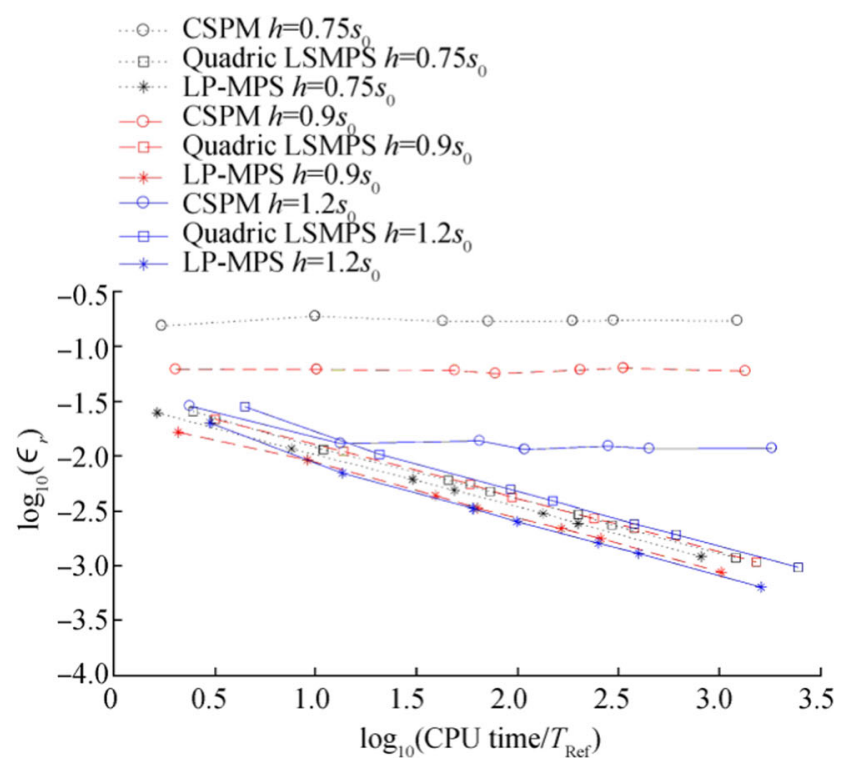

(b) CSPM, Quadric LSMPS, LP-MPS

Figure 4 Relative error of Laplacian discretisation vs CPU time for estimating Laplacian of $p(x, y)=x^{6} y^{6}$ in the cases with different ratios $h / s_{\mathbf{0}}\left(K=0.4 ; T_{\text {Ref }}\right.$ is the CPU time spent by CSPH2 $\Gamma$ with $s_{0}=0.1$ and $h=0.75 s_{0}$; error estimation domain $2.25<x<2.75,2.25<y<2.75$ ). a CSPH2 $\Gamma$, ICSPM, QSFDI. b CSPM, Quadric LSMPS, LP-MPS

random distribution with different values of $K$. The relative error in the tests is defined by

$E_{r}=\sqrt{\sum_{i=1}^{N_{t}}\left(\nabla^{2} p_{i}-\left\langle\nabla^{2} p_{i}\right\rangle\right)^{2}} / \sqrt{\sum_{i=1}^{N_{t}}\left(\nabla^{2} p_{i}\right)^{2}}$

where $N$ is the number of particles for error estimations to be consistent with the references. Similar to the cases shown in Figures 1, 2, and 3, the relative error at inner particles away from boundaries, i.e. $0.25 \leq x \leq 0.75 ; 0.25 \leq y \leq 0.75$, is 


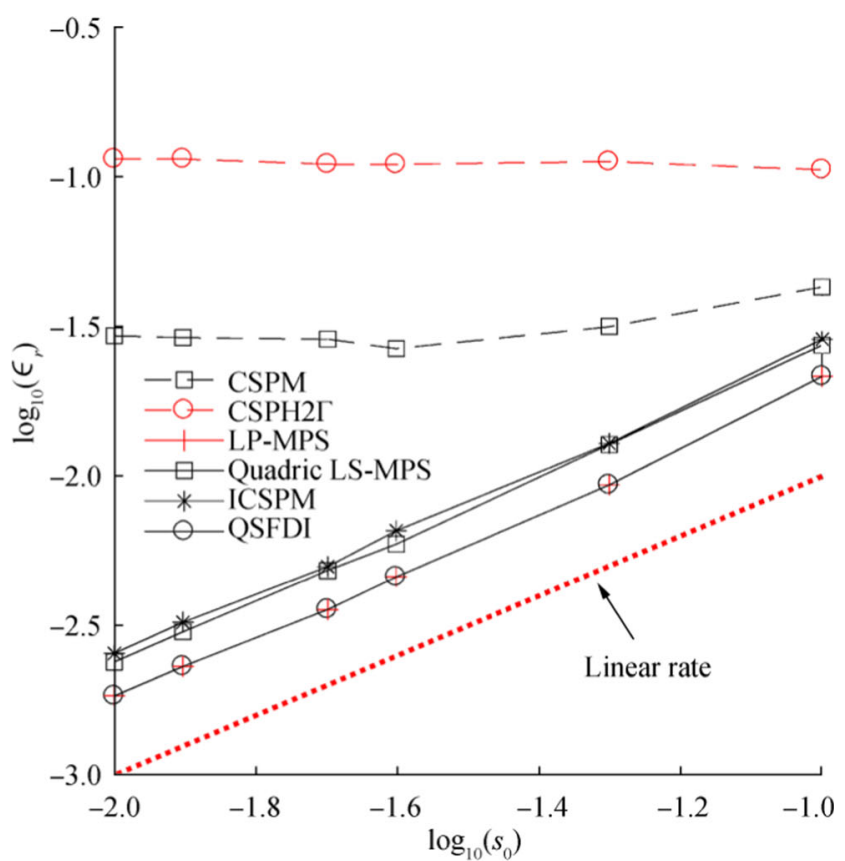

(a) $K=0.6$

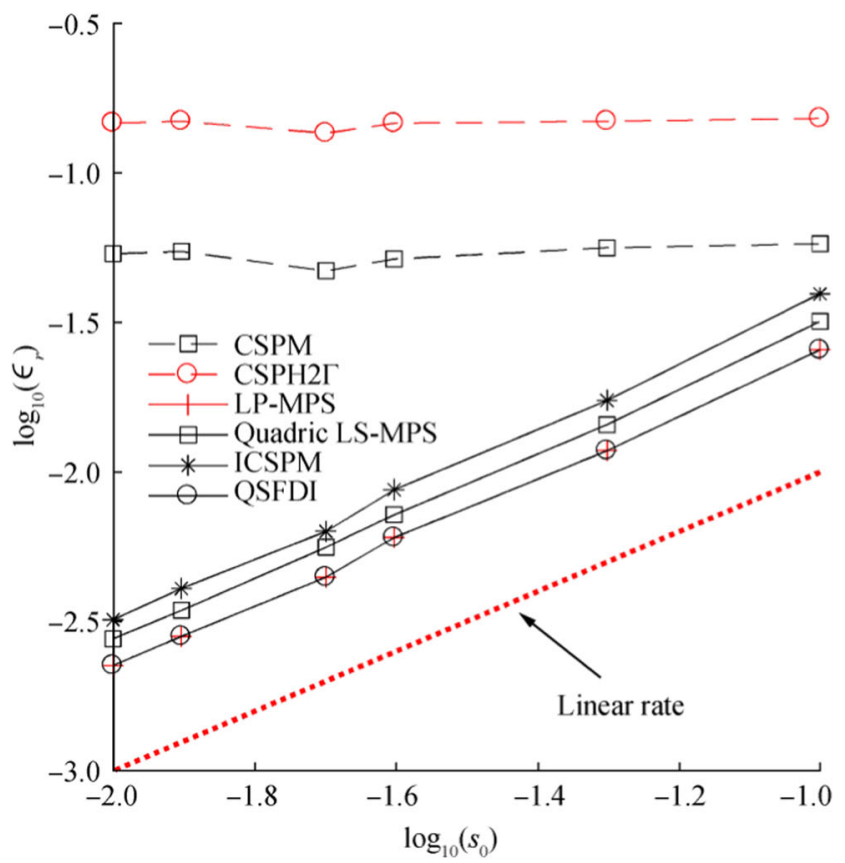

(b) $K=0.8$

Figure 5 Relative error for estimating Laplacian of $p(x, y)=x^{6} y^{6}$ for $K=0.6$ and $K=0.8\left(h=1.2 s_{0}\right.$ for CSPM, CSPH2 $\Gamma$ and ICSPM, $h=0.75 s_{0}$ for other schemes; the slopes of the dotted line is 1 ; error estimation domain $2.25<x<2.75,2.25<y<2.75$ )

assessed. In addition, to reflect the overall performances of different schemes at both the inner particles and boundary particles, the relative error estimated by considering all

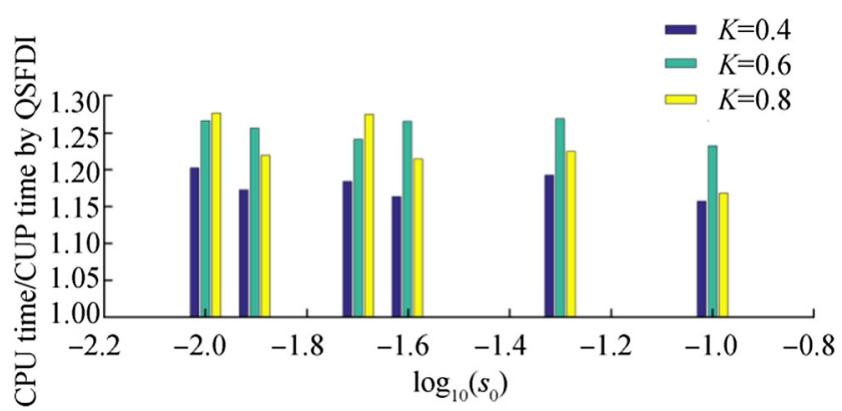

Figure 6 Ratio of the CPU time spent by the LP-MPS and that by the QSFDI on estimating Laplacian of $p(x, y)=x^{6} y^{6}\left(h=0.75 s_{0}\right)$ for the cases with different randomness of particle distribution

particles are also assessed.

Figure 7 displays the relative errors for estimating Laplacians of Eqs. (12) and (13) in the cases with $K$ $=0.4$. For clarity, the corresponding results by ICSPM are not shown. From Figure $7 \mathrm{a}$, c, in which only inner particles are taken into account when estimating the relative error, it is observed that all schemes converge at a rate between linear and quadric for relatively coarse particle resolutions, i.e. $s_{0}>0.01$; thereafter, the relative errors of the CSPM and CSPH2 $\Gamma$ seem not to be reduced, whereas the QSFDI, the LP-MPS and quadric LS-MPS converge at a rate slightly higher than a linear rate. As expected, for a specific particle resolution, the relative errors of all the schemes considering all particles (Figure 7b, d) including these on boundaries are relatively higher than the corresponding results considering the inner particles only. The CSPM and CSPH2 $\Gamma$ converge at a rate much lower than a linear rate (the mean slopes of the curves for CSPM and CSPH2 $\Gamma$ are about 1/0.5). The QSFDI, the LP-MPS and quadric LSMPS converge at a rate with the mean slopes of approximately $1 / 1.5$.

The same conclusions are achieved in the cases with other randomness, e.g. Figure 8, which displays the relative errors considering all particles for estimating Laplacian of Eqs. (12) and (13), respectively, obtained by using $K=0.8$. Examination of the corresponding robustness is also carried out for these cases. The CPU time for different accuracy corresponding to the results of Figure 8 is illustrated in Figure 9. Once again, the superiority of the QSFDI over other schemes in terms of the accuracy and computational costs is clearly observed as in Figures 3 and 6.

\subsection{Finding solutions of Poisson's equation}

Another purpose of the Laplacian discretisation is to discretise the governing equation, e.g. the Poisson's equation, to find its numerical solution. Relevant patch tests presented in this 
Figure 7 Relative error for estimating Laplacians of Eq. (12) and (13) $\left(K=0.4 ; h=1.2 s_{0}\right.$ for CSPM and CSPH $2 \Gamma, h=0.9 s_{0}$ for other schemes; internal particles located at $2.25<x<2.75,2.25<y$ $<2.75$ are used for the error estimation in (a) and (c))

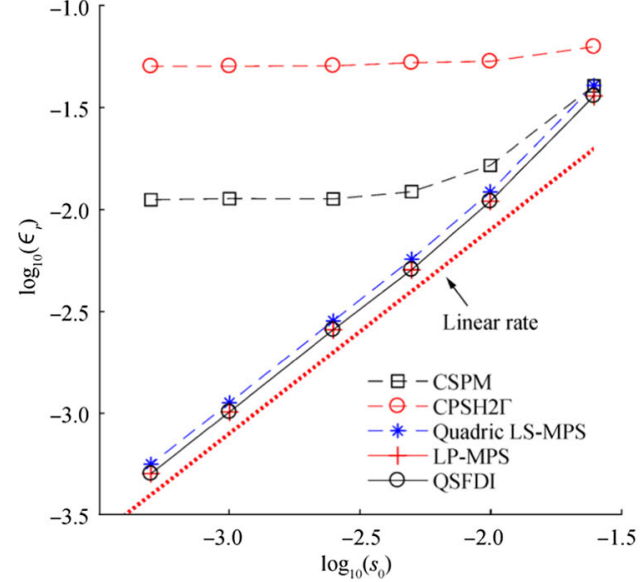

(a) Internal particle Eq. (12)

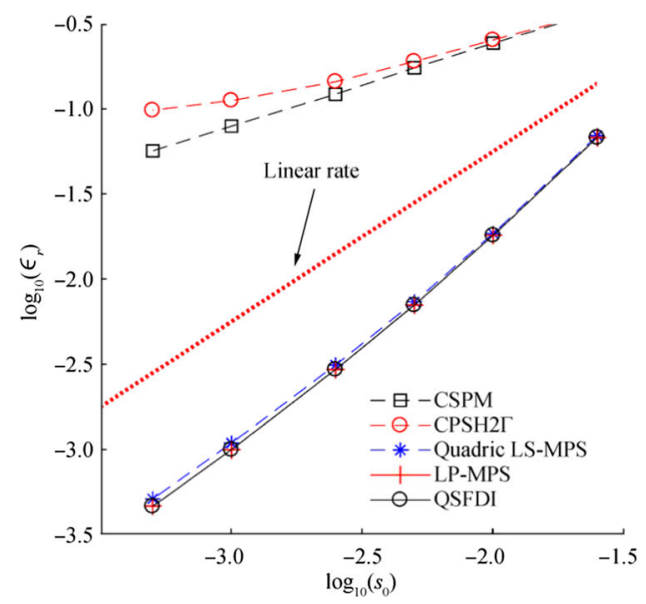

(b) All particle Eq. (12)

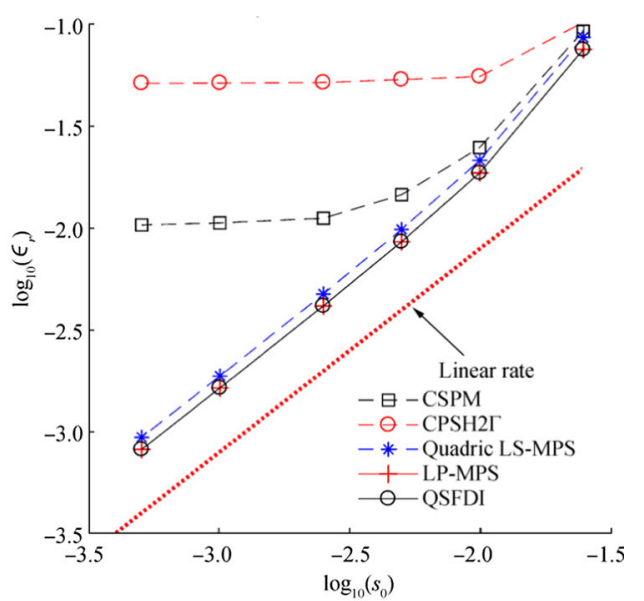

(c) Internal particle Eq. (13)

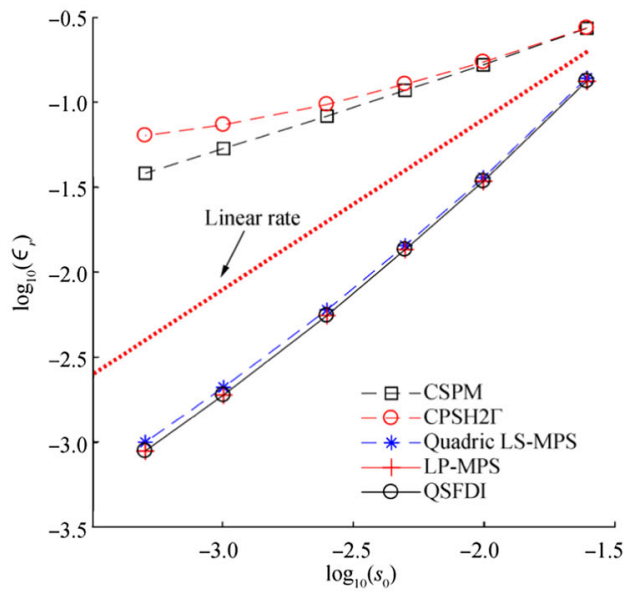

(d) All particle Eq. (13) section will use a computational domain of a unit square $(0 \leq x \leq 1,0 \leq y \leq 1)$, the same as that for Figures 7, 8, and 9 . In this test, the Poisson's equation defined by Eq. (8), of which (R. H.S) are specified by the Laplacians of a given function. The Dirichlet condition on all boundaries of the domain is applied, i.e. $p$ at all boundary particles are specified to be consistent with (R.H.S). Under this condition, the analytical solution of the Poisson's equation (Eq. (8)) in the computational domain is the function $p(x, y)$. For example, if Eq. (13) is applied as the function $p(x, y),(R . H . S)=-100 \sin (6 x+8 y)$ and the values on the boundary at $x=1.0$ is $\sin (6+8 y)$. The Poisson's equation is discretised at all internal particles by using different schemes to form linear algebraic equations, which is solved by the GMRESS solver, resulting in the solutions of $p(x, y)$ at all internal particles. For the results shown below, the control residual adopted by the GMRESS solver is $10^{-4}$, which is sufficiently small (comparison with the corresponding results obtained using a control residual of $10^{-8}$ shows that the difference is smaller than $0.1 \%$ ). The relative error of the numerical solution against the analytical solution is evaluated by

$\varepsilon_{r}=\sqrt{\sum_{i=1}^{N_{t}}\left|p_{i}-p_{i, a}\right|^{2}} / \sqrt{\sum_{i=1}^{N_{t}}\left|p_{i, \mathrm{a}}\right|^{2}}$

where $p_{i}$ and $p_{i, a}$ are the numerical approximation and the analytical value, respectively, at particle $i ; N_{t}$ is the total number of internal particles.

Figure 10 compares the relative errors of numerical solutions to the Poisson's equations, whose $(R . H . S)$ are given by the Laplacian of Eqs. (12) and (13), respectively, with the moderate randomness of particle distribution $(K=0.4)$. It is observed that the convergent rates of the LP-MPS, quadric LS-MPS, ICSPM and the QSFDI are quadric for all particle spacing. In contrast, as $s_{0}$ decreases, the relative errors of the CSPM and CSPH $2 \Gamma$ reduces at a quadric rate for relatively coarse resolutions (when the error is large); however, it reduces to a linear or lower rate for finer particle resolutions 


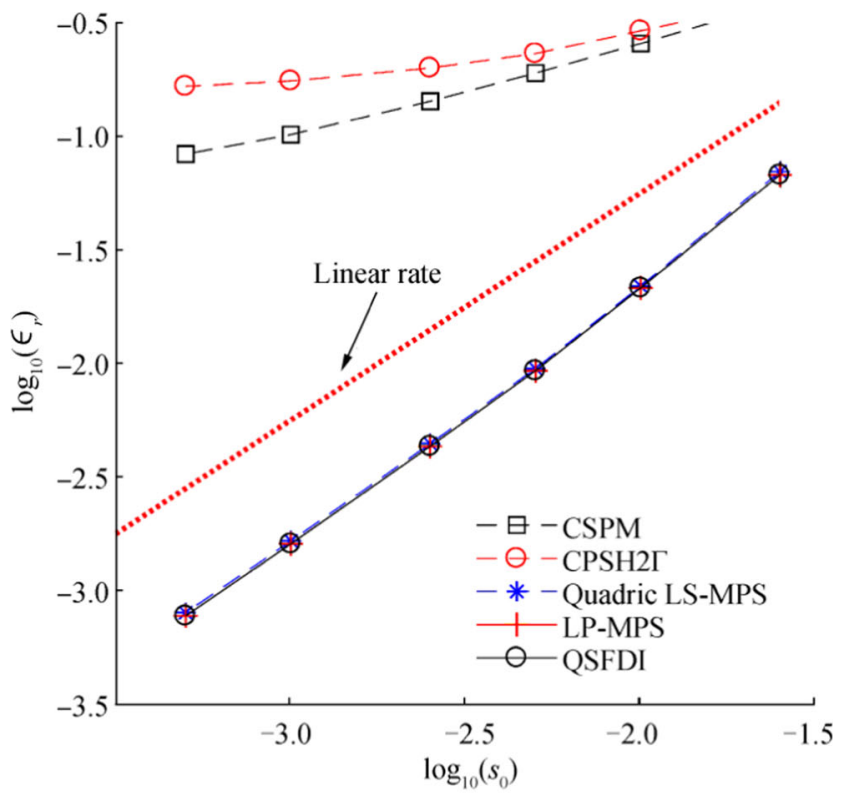

(a) All particle Eq. (12)

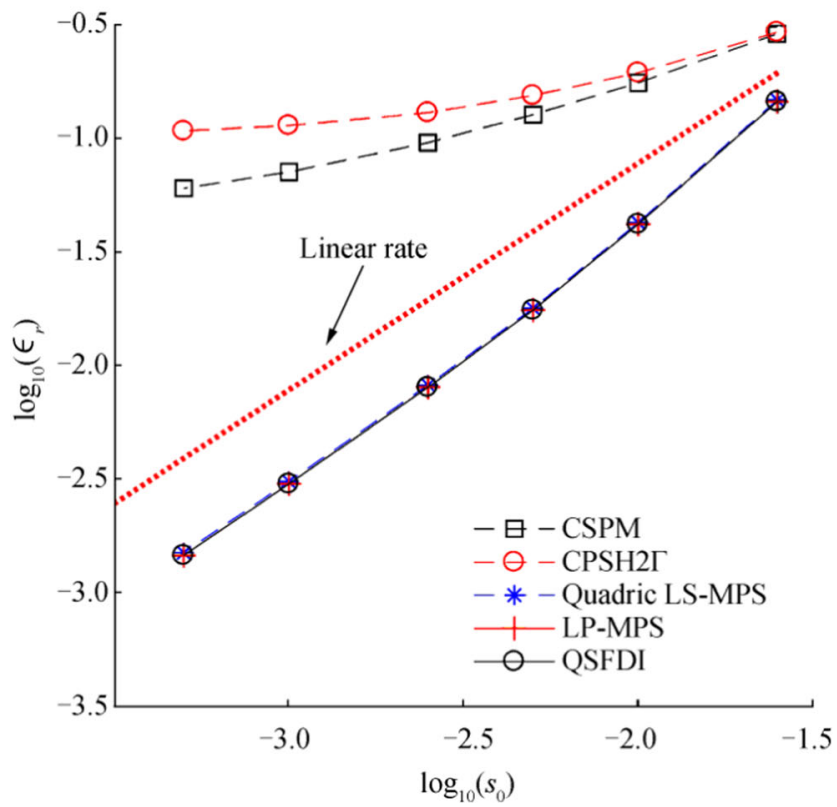

(b) All particle Eq. (13)

Figure 8 Relative error for estimating Laplacians of Eqs. (12) and (13) at all particles $\left(K=0.8 ; h=1.2 s_{0}\right.$ for CSPM and CSPH2 $\Gamma, h=0.9 s_{0}$ for other schemes

(when the error becomes acceptably small). The comparison of the relative errors for a specific particle spacing indicates that the QSFDI and the LP-MPS result in the most accurate solutions. The corresponding comparisons of the CPU time are illustrated in Figure 11. Unlike the direct Laplacian discretisation presented in Section 3.1, the CPU time spent on achieving the solutions to the Poisson's equation is also influenced by the effectiveness of the linear algebraic solver

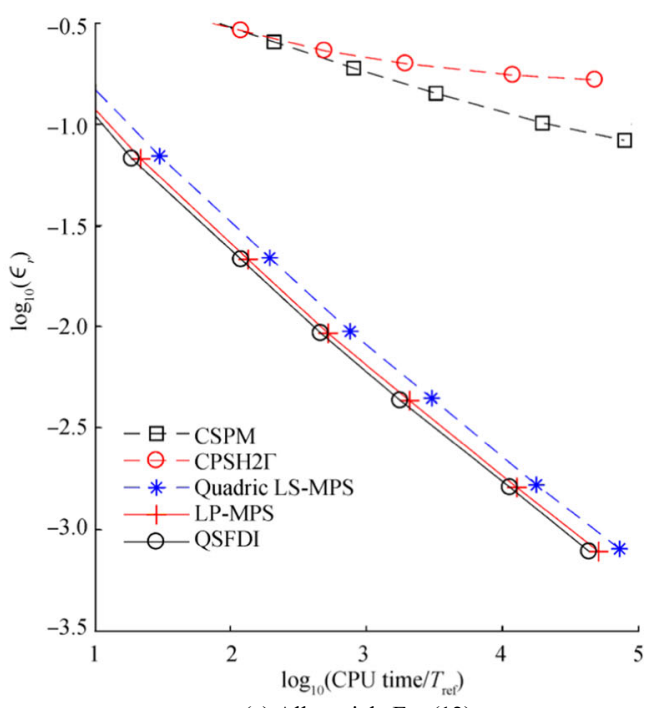

(a) All particle Eq. (12)

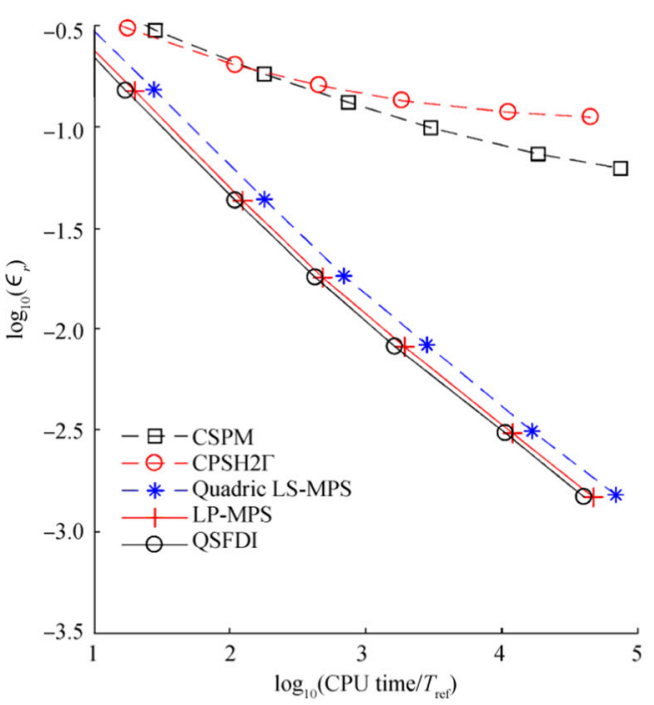

(b) All particle Eq. (13)

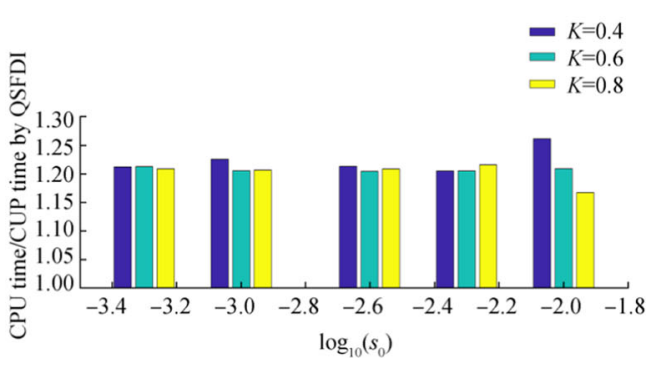

(c) Ratio of CPU time by the LP-MPS against that by the QSFDI

Figure 9 CPU times for estimating Laplacians of Eq. (12) and (13) $(K=$ $0.8 ; h=1.2 s_{0}$ for CSPM and CSPH2 $\Gamma, h=0.9 s_{0}$ for other schemes; $T_{\mathrm{Ref}}$ is the CPU time spent by CSPH2 $\Gamma$ with $s_{0}=0.1$ )

and its pre-conditioner, i.e. the initial value. By using the solver briefed above, the total CPU time spent by the QSFDI is slightly shorter than the LP-MPS but significantly shorter than all other schemes. 


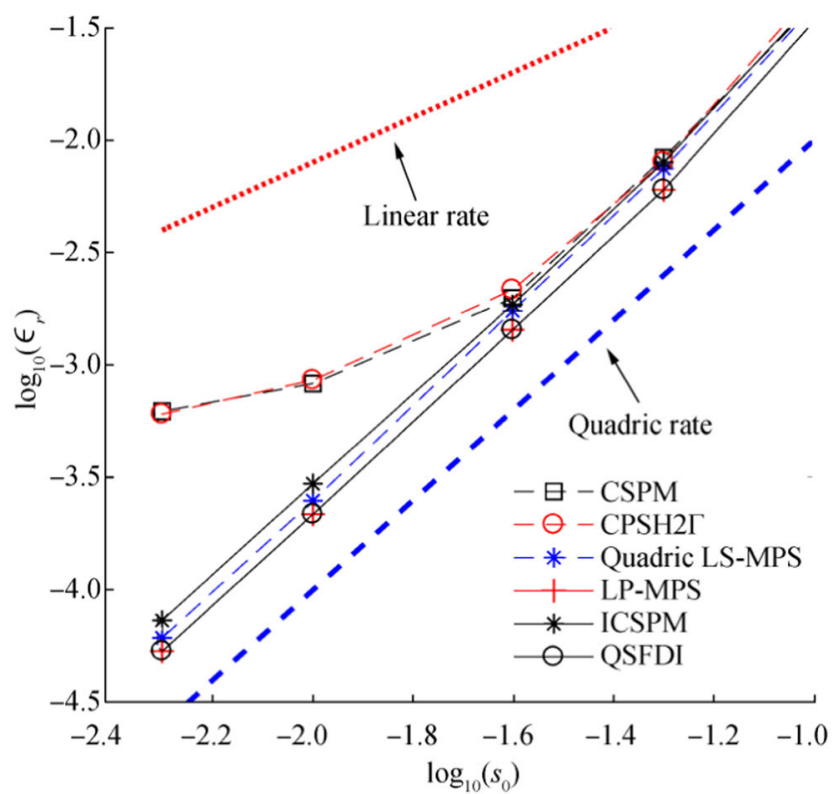

(a) Eq. (12)

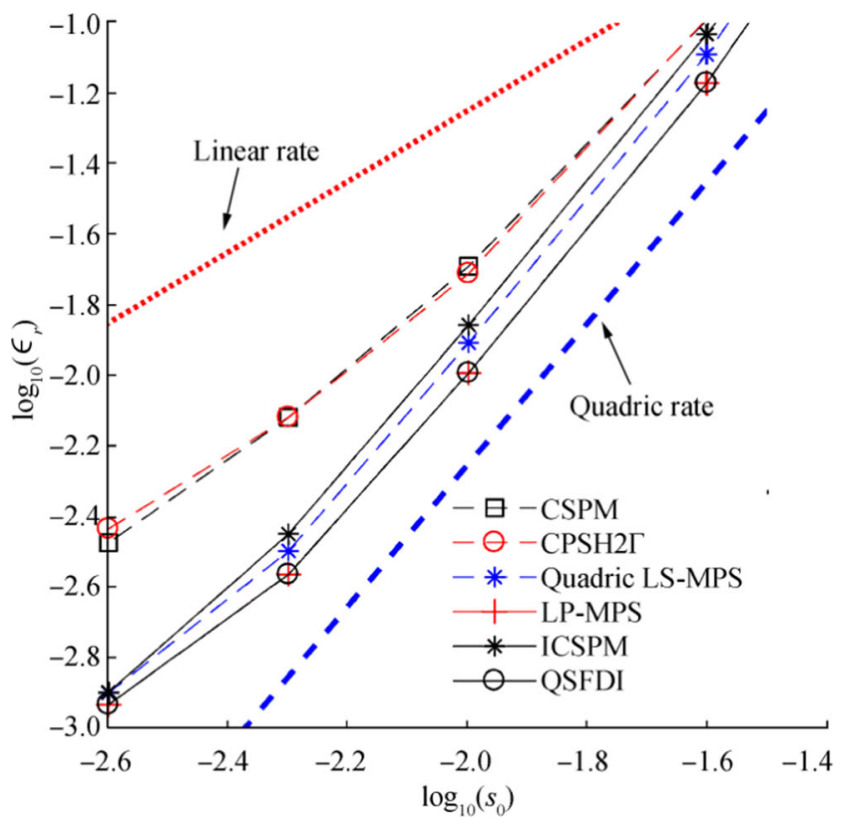

(b) Eq. (13)

Figure 10 Relative error of solution to Poisson's equation based on Eq. (12) and Eq. (13) $\left(K=0.4, h=1.2 s_{0}\right.$ for CSPM, CSPH2 $\Gamma$ and ICSPM, $h=0.9 s_{0}$ for other schemes)

Different values of $K$ and $h$ are also used in this investigation. Some results are illustrated in Figures 12 and 13 for $K=$ 0.6 and 0.8 respectively, where $h=1.2 s_{0}$ are used for all schemes. For clarity, the corresponding results with the quadric LP-MPS and the ICSPM are not shown. As can be seen, with severer randomness of particle distribution, the relative errors of the $\mathrm{CSPM}$ and $\mathrm{CSPH} 2 \Gamma$ reduce at a rate less than the linear rate

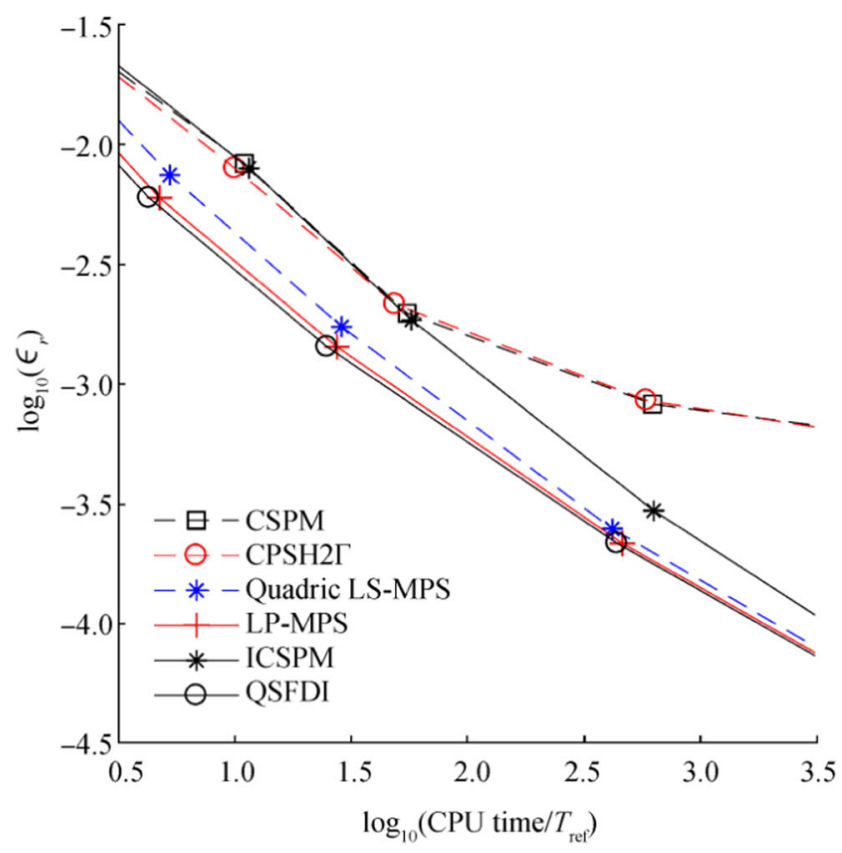

(a) Eq. (12)

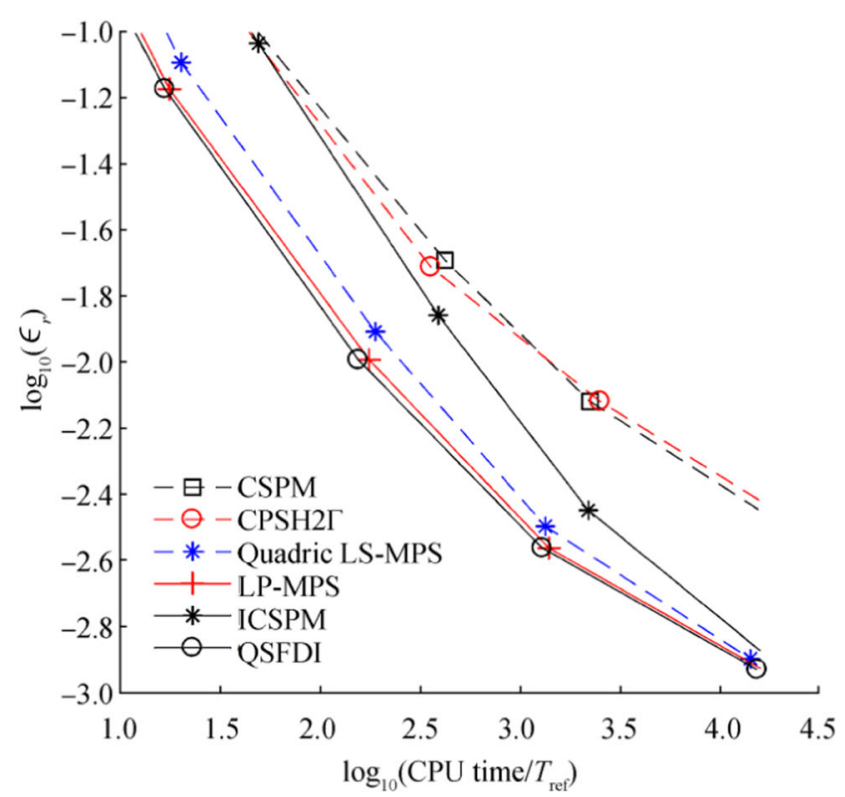

(b) Eq. (13)

Figure $11 \mathrm{CPU}$ times for solving Poisson's equation based on Eqs. (12) and (13) $\left(K=0.4, h=1.2 s_{0}\right.$ for CSPM, CSPH2 $\Gamma$ and ICSPM, $h=0.9 s_{0}$ for other schemes)

as $s_{0}$ decreases, quite different from what has been seen in Figure 10. In contrast, the accuracy and convergent properties of the QSFDI and LP-MPS seem to be insignificantly affected by increasing the randomness (Figures 12a, c and 13a, c). Figures $12 \mathrm{~b}$ and $\mathrm{d}$ and $13 \mathrm{~b}$ and $\mathrm{d}$ reveal that the CPU time by the present QSFDI is generally shorter than all other schemes for achieving satisfactory results, e.g. relative error smaller than 
Figure 12 Relative error of solution to Poisson's equation based on Eq. (12) and the corresponding CPU time in the cases with different particle randomness $\left(h=1.2 s_{0} ; T_{\text {Ref }}\right.$ is the CPU time spent by $\mathrm{CSPH} 2 \Gamma$ with $s_{0}=0.1$ and $K=0.6$ )

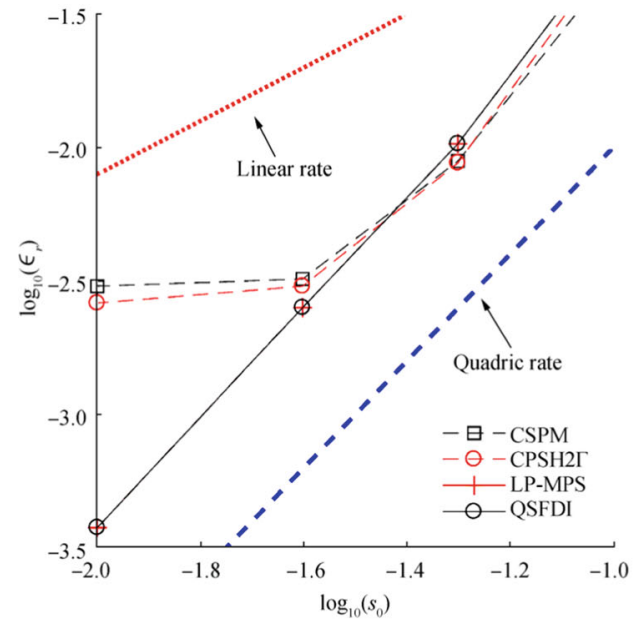

(a) $\epsilon_{r}$ vs $s_{0}, K=0.6$

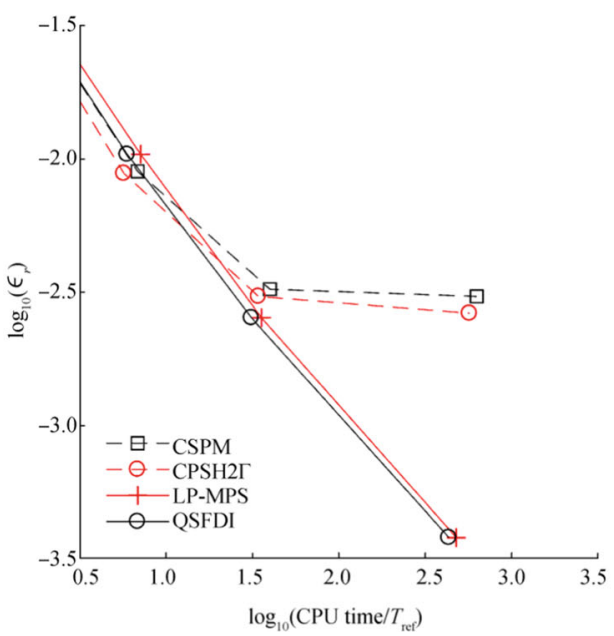

(b) $\epsilon_{r}$ vs CPU time, $K=0.6$

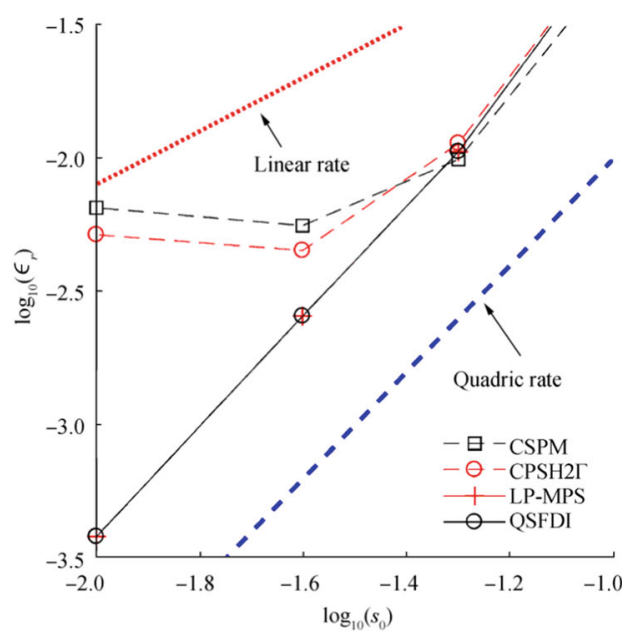

(c) $\epsilon_{r}$ vs $s_{0}, K=0.8$

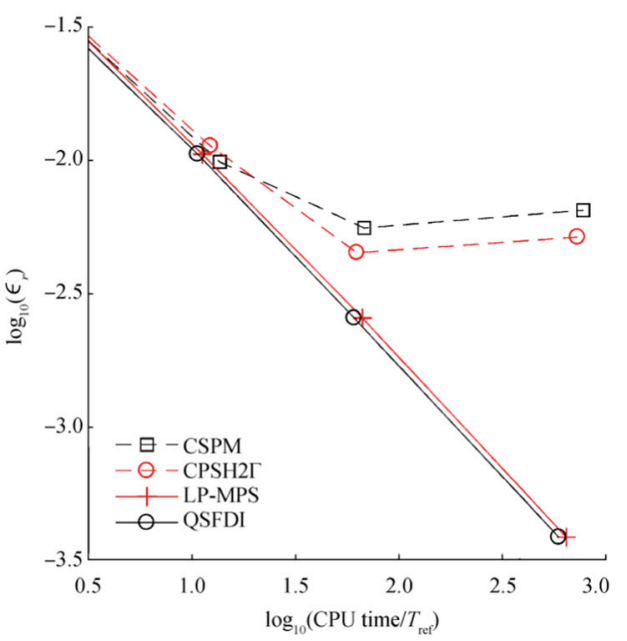

(d) $\epsilon_{r}$ vs CPU time, $K=0.6$
$1 \%$. Following the comparison of the robustness of the QSFDI and the LP-MPS in the previous section on estimating Laplacians, the average ratios of the CPU time spent by the LP-MPS and that by the QSFDI are displayed in Figure 14, where $h=1.2 s_{0}$, for finding the solutions to the Poisson's equation based on Eq. (13). It clearly shows that the CPU time spent by the QSFDI is approximately 5\%-10\% shorter than the LPMPS, although averagely $20 \%$ less CPU time on discretising the Poisson's equation than that by the QSFDI is recorded in the patch tests in Section 3.1.

\section{Conclusions}

This paper develops a new scheme called QSFDI, which adopts the same principle of SFDI, to discretise the Laplacian operator for Lagrangian meshless (particle) methods, in which the particles move during the numerical simulation and exhibit a disordered/random distribution. The accuracy and consistency of the QSFDI are similar to the LSMPS and LP-MPS but higher than the CSPM and CSPH for randomly distributed particles. However, the matrices required to be inversed by the QSFDI have smaller sizes than the LSMPS and LP-MPS. For example, for 3D problems, the size of the matrices to be inversed in the QSFDI is $3 \times 3$, while it is $6 \times 6$ in LP-MPS.

Systematic patch tests considering both directly estimating the Laplacian of specific functions and solving Poisson's equations are carried out. In these tests, different functions including polynomials, hyperbolic and trigonometric functions, which may represent typical spatial variations of physical quantities in engineering such as the water waves and the thermodynamics, are considered. The particles used in the patch tests are randomly distributed. It is observed that the 
Figure 13 Relative error of solution to Poisson's equation based on Eq. (13) and the corresponding CPU time in the cases with increased particle randomness $\left(h=1.2 s_{0} ; T_{\text {Ref }}\right.$ is the CPU time spent by CSPH $2 \Gamma$ with $s_{0}=0.1$ and $K=0.6$ )

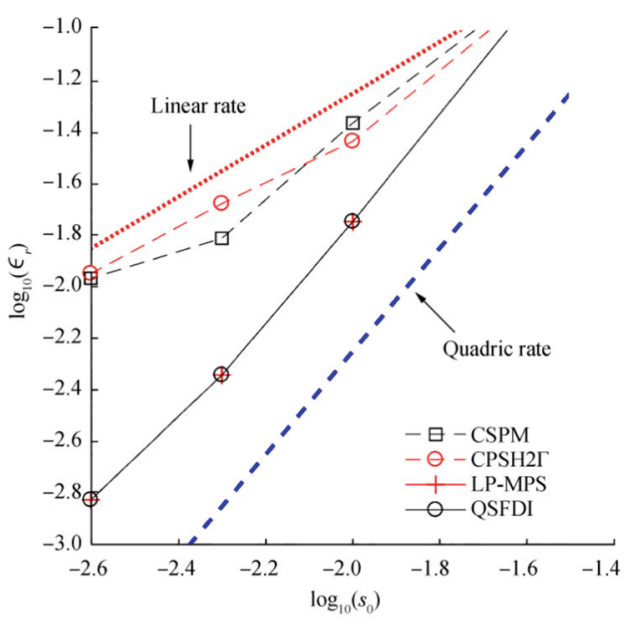

(a) $\epsilon_{r}$ vs $s_{0}, K=0.6$

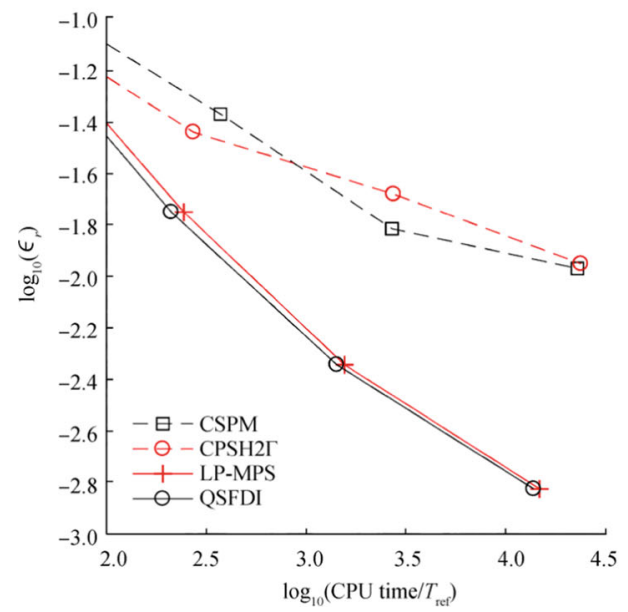

(b) $\epsilon_{r}$ vs CPU time, $K=0.6$

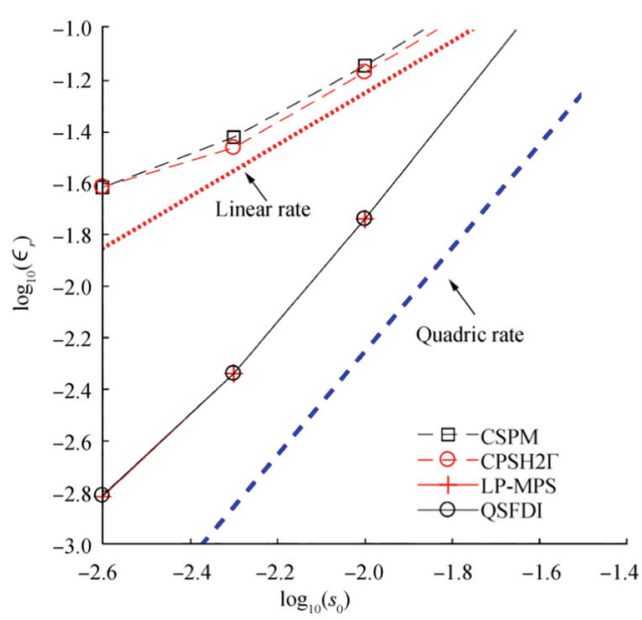

(c) $\epsilon_{r}$ vs $s_{0}, K=0.8$

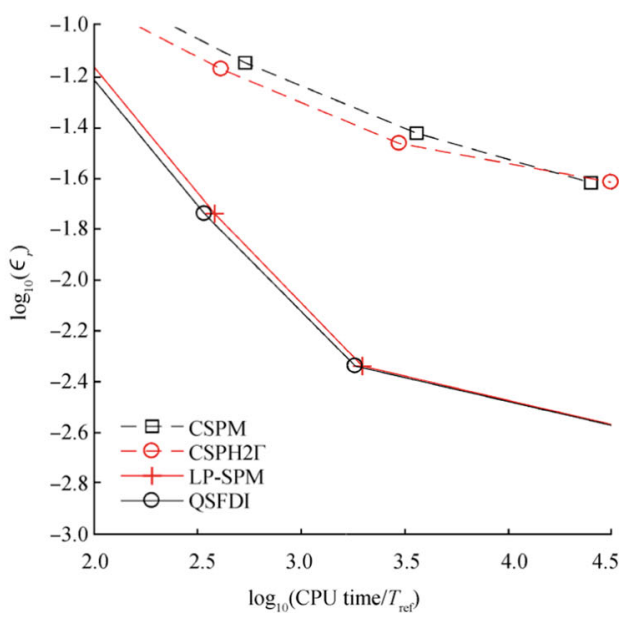

(d) $\epsilon_{r}$ vs CPU time, $K=0.8$
QSFDI has the same convergent rate as the LP-MPS and quadric LSMPS, which is higher than that of the CSPM and $\mathrm{CSPH}$ in all the cases studied. It is also observed that the

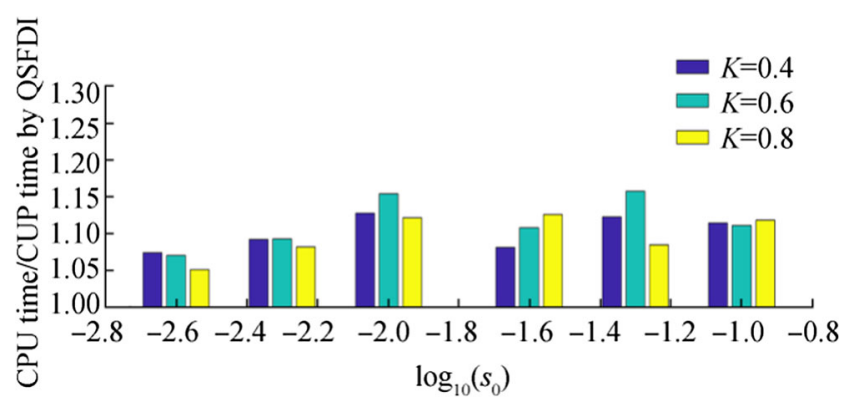

Figure 14 Average ratio of the CPU time spent by the LP-MPS against that by the QSFDI in the cases with different randomness for finding solutions to Poisson's equation ( $\left.h=1.2 s_{0}\right)$
QSFDI requires considerably less computational time than all other schemes (such as the LP-MPS) to achieve the same accuracy.

It is worth noting that the QSFDI method presented in the paper does not only give a new formula for the Laplacian discretisation but also provides the new schemes for the numerical interpolation and gradient estimations. This means that one may extend the QSFDI to deal with the first and $2^{\text {nd }}$ derivatives in differential equations, e.g. the NS equation and advection-diffusion equations, with a linear consistency and quadric accuracy.

It shall be also noted that the implementation of the QSFDI in a Lagrangian meshless method to solve engineering problems, such as wave-structure interaction in maritime engineering, is under study and results will be discussed in other publications. 


\section{Appendix 1: Derivation of QSFDI}

For each particle $j$ at $\boldsymbol{x}_{j}$, which locates inside the support domain $\Omega_{I}$ of point $\boldsymbol{x}_{I}$, a function $p$ can be expressed as Taylor's expansion, i.e. Eq. (1). Multiplying Eq. (1) by $w_{j I} \boldsymbol{r}_{j I}^{(2 c)} / d_{j I}^{4}$, where $w_{j I}$ is the weighting function for particle $j$ related to $\boldsymbol{x}_{I}, d_{j I}$ is the distance between particle $j$ and $\boldsymbol{x}_{I}$, ignoring the truncation error and taking the sum of resultant equations for all particles in $\Omega_{I}$, it yields

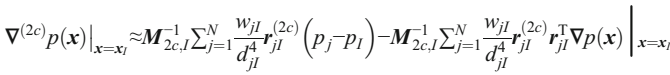

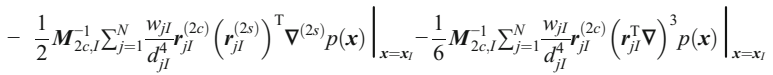

in which $\boldsymbol{M}_{2 c, I}=\sum_{j=1}^{N} \frac{w_{j I}}{d_{j I}^{4}} \boldsymbol{r}_{j I}^{(2 c)}\left(\boldsymbol{r}_{j I}^{(2 c)}\right)^{\mathrm{T}}$. For convenience, $\left.\left(\boldsymbol{r}_{j I}^{\mathrm{T}} \boldsymbol{\nabla}\right){ }^{3} p(\boldsymbol{x})\right|_{\boldsymbol{x}=\boldsymbol{x}_{I}}$ is re-written as $\left.\left(\boldsymbol{r}_{j I}^{(3)}\right)^{\mathrm{T}} \nabla^{(3)} p(\boldsymbol{x})\right|_{\boldsymbol{x}=\boldsymbol{x}_{I}}$, where $\boldsymbol{r}_{j I}^{(3)}=\left[\begin{array}{lllllll}x_{j I}^{3} & 3 x_{j I}^{2} y_{j I} & 3 x_{j I}^{2} z_{j I} & 3 x_{j I} y_{j I}^{2} & 6 x_{j I} y_{j j} z_{j I} & 3 x_{j I} z_{j I}^{2} & y_{j I}^{3}\end{array}\right.$

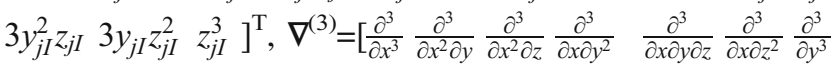
$\left.\frac{\partial^{3}}{\partial y^{2} \partial z} \frac{\partial^{3}}{\partial y \partial z^{2}} \frac{\partial^{3}}{\partial z^{3}}\right]^{\mathrm{T}}$ are two $10 \times 1$ matrices. Substituting Eq. (16) into Eq. (1), it leads to

$$
\begin{gathered}
p_{j}-p_{I} \approx\left(\boldsymbol{r}_{j I}^{(2 c)}\right)^{\mathrm{T}} \boldsymbol{M}_{2 c, I}^{-1} \sum_{k=1}^{N} \frac{w_{k I}}{d_{k I}^{4}} \boldsymbol{r}_{k I}^{(2 c)}\left(p_{k}-p_{I}\right)+\left.\boldsymbol{G}_{j I}^{\mathrm{T}} \nabla p(\boldsymbol{x})\right|_{\boldsymbol{x}=\boldsymbol{x}_{I}} \\
+\left.\frac{1}{2} \boldsymbol{\Pi}_{j I}^{\mathrm{T}} \boldsymbol{\nabla}^{(2 s)} p(\boldsymbol{x})\right|_{\boldsymbol{x}=\boldsymbol{x}_{I}}+\left.\frac{1}{6} \boldsymbol{F}_{j I}^{\mathrm{T}} \boldsymbol{\nabla}^{(3)} p(\boldsymbol{x})\right|_{\boldsymbol{x}=\boldsymbol{x}_{I}}
\end{gathered}
$$

where

$$
\begin{gathered}
\boldsymbol{\Pi}_{j I}=\left\{\left(\boldsymbol{r}_{j I}^{(2 s)}\right)^{\mathrm{T}}-\left(\boldsymbol{r}_{j I}^{(2 c)}\right)^{\mathrm{T}} \boldsymbol{M}_{2 c, I}^{-1} \sum_{k=1}^{N} \frac{w_{k I}}{d_{k I}^{4}} \boldsymbol{r}_{k I}^{(2 c)}\left(\boldsymbol{r}_{j I}^{(2 s)}\right)^{\mathrm{T}}\right\}^{\mathrm{T}}, \\
\boldsymbol{G}_{j I}=\left\{\boldsymbol{r}_{j I}^{\mathrm{T}}-\left(\boldsymbol{r}_{j I}^{(2 c)}\right)^{\mathrm{T}} \boldsymbol{M}_{2 c, I}^{-1} \sum_{k=1}^{N} \frac{w_{k I}}{d_{k I}^{4}} \boldsymbol{r}_{k I}^{(2 c)} \boldsymbol{r}_{k I}^{T}\right\}^{\mathrm{T}}, \\
\boldsymbol{F}_{j I}=\left\{\left(\boldsymbol{r}_{j I}^{(3)}\right)^{\mathrm{T}}-\left(\boldsymbol{r}_{j I}^{(2 c)}\right)^{\mathrm{T}} \boldsymbol{M}_{2 c, I}^{-1} \sum_{k=1}^{N} \frac{w_{k I}}{d_{k I}^{4}} \boldsymbol{r}_{k I}^{(2 c)}\left(\boldsymbol{r}_{k I}^{(3)}\right)^{\mathrm{T}}\right\}^{\mathrm{T}} .
\end{gathered}
$$

Multiplying Eq. (17) by $w_{j I} \boldsymbol{\Pi}_{j I} / d_{j I}^{4}$ and taking the sum of resultant equations for all particles in $\Omega_{I}$, it leads to

$$
\begin{aligned}
& \left.\nabla^{(2 s)} p(\boldsymbol{x})\right|_{x=x_{I}} \approx 2 \boldsymbol{M}_{2 s, I}^{1} \sum_{j=1}^{N} \boldsymbol{\Gamma}_{j I}\left(p_{j}-p_{I}\right)-\left.2 \boldsymbol{M}_{2 s, I}^{-1} L_{j=1}^{N} \frac{w_{j I}}{d_{j I}^{4}} \boldsymbol{\Pi}_{j l} \boldsymbol{G}_{j I}^{\mathrm{T}} \nabla p(\boldsymbol{x})\right|_{x=x_{I}} \\
& -\left.\frac{1}{3} \boldsymbol{M}_{2 s, I}^{-1} \sum_{j=1}^{N} \frac{w_{j I}}{d_{j I}^{4}} \Pi_{j I} \boldsymbol{F}_{j I}^{\mathrm{T}} \boldsymbol{\nabla}^{(3)} p(\boldsymbol{x})\right|_{x=\boldsymbol{x}_{I}}
\end{aligned}
$$

where

$$
\boldsymbol{M}_{2 s, I}=\sum_{j=1}^{N} \frac{w_{j l} I}{d_{j l}^{4}} \Pi_{j I} \Pi_{j I}^{\mathrm{T}} \operatorname{and}_{j I}=\left(\frac{w_{j I}}{d_{j I}^{4}} \boldsymbol{\Pi}_{j I}-\boldsymbol{\Pi}_{j I}\left(r_{j I}^{(2 c)}\right)^{\mathrm{T}} \boldsymbol{M}_{2 c, I}^{-1} \sum_{k=1}^{N} \frac{w_{k I}}{d_{k I}^{4}} \boldsymbol{r}_{k I}^{(2 c)}\right) .
$$

Substituting Eq. (18) to Eq. (17) leads to

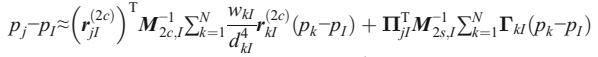

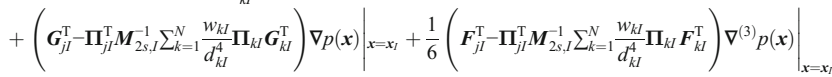

Multiplying Eq. (19) by $w_{j I} \boldsymbol{q}_{j I} / d_{j I}^{2}$, where

$\boldsymbol{q}_{j I}=\left(\boldsymbol{G}_{j I}^{\mathrm{T}}-\boldsymbol{\Pi}_{j I}^{\mathrm{T}} \boldsymbol{M}_{2 s, I}^{-1} \sum_{k=1}^{N} \frac{w_{k I}}{d_{k I}^{4}} \boldsymbol{\Pi}_{k I} \boldsymbol{G}_{k I}^{\mathrm{T}}\right)^{\mathrm{T}}$,

and taking the sum of resultant equations for all particles in $\Omega_{I}$, it leads to the formula for approximating the gradient, i.e. $\left\langle\left.\nabla p(\boldsymbol{x})\right|_{\boldsymbol{x}=\boldsymbol{x}_{I}}\right\rangle$, and its leading truncation error, $\boldsymbol{E}_{\left.\nabla p(\boldsymbol{x})\right|_{x=x_{I}}}$,

$$
\begin{gathered}
\left\langle\left.\nabla p(\boldsymbol{x})\right|_{x=x_{I}}\right\rangle=\boldsymbol{M}_{1 q, I}^{-1} \sum_{j=1}^{N} \frac{w_{j I}}{d_{j I}^{2}} \boldsymbol{q}_{j I}\left(p_{j}-p_{I}\right)-\boldsymbol{M}_{1 q, I}^{-1} \sum_{j=1}^{N} \frac{w_{j I}}{d_{j I}^{2}} \boldsymbol{q}_{j I}\left(\boldsymbol{r}_{j I}^{(2 c)}\right)^{\mathrm{T}} \\
\boldsymbol{M}_{2 c, I}^{-1} \sum_{k=1}^{N} \frac{w_{k I}}{d_{k I}^{4}} \boldsymbol{r}_{k I}^{(2 c)}\left(p_{k}-p_{I}\right)-\boldsymbol{M}_{1 q, I}^{-1} \sum_{j=1}^{N} \frac{w_{j I}}{d_{j I}^{2}} \boldsymbol{q}_{j I} \boldsymbol{\Pi}_{j I}^{\mathrm{T}} \boldsymbol{M}_{2 s, I}^{-1} \sum_{k=1}^{N} \boldsymbol{\Gamma}_{k I}\left(p_{k}-p_{I}\right)
\end{gathered}
$$

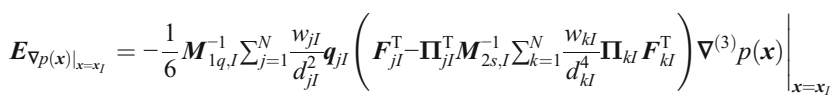

where \langle\rangle indicates an approximated value and

$\boldsymbol{M}_{1 q, I}=\sum_{j=1}^{N} \frac{w_{j I}}{d_{j I}^{2}} \boldsymbol{q}_{j I} \boldsymbol{q}_{j I}^{\mathrm{T}}$.

Substituting Eq. (20) to Eq. (18), it leads to the formula to approximate the $\left.\boldsymbol{\nabla}^{(2 s)} p(\boldsymbol{x})\right|_{\boldsymbol{x}=\boldsymbol{x}_{I}}$, i.e.,

$$
\left\langle\left.\nabla^{(2 s)} p(\boldsymbol{x})\right|_{\boldsymbol{x}=x_{l}}\right\rangle=2 \boldsymbol{M}_{2 s, I}^{-1} \sum_{j=1}^{N} \boldsymbol{\Gamma}_{j I}\left(p_{j}-p_{I}\right)-2 \boldsymbol{M}_{2 s, I}^{-1} \sum_{j=1}^{N} \frac{w_{j I}}{d_{j l}^{4}} \Pi_{j l} \boldsymbol{G}_{j I}^{\mathrm{T}}\left\langle\left.\nabla p(\boldsymbol{x})\right|_{\boldsymbol{x}=\boldsymbol{x}_{l}}\right\rangle
$$

with its leading truncation error

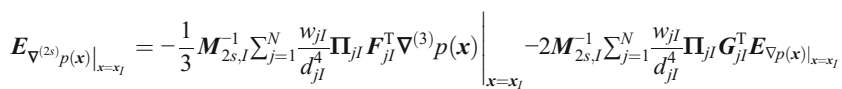

The Laplacian can therefore be approximated by using

$$
\left\langle\left.\nabla^{2} p(\boldsymbol{x})\right|_{\boldsymbol{x}=\boldsymbol{x}_{I}}\right\rangle=\boldsymbol{I}^{\mathrm{T}}\left\langle\left.\nabla^{(2 s)} p(\boldsymbol{x})\right|_{\boldsymbol{x}=\boldsymbol{x}_{I}}\right\rangle
$$


where $\boldsymbol{I}=\left[\begin{array}{lll}1 & 1 & 1\end{array}\right]^{\mathrm{T}}$. The corresponding leading truncation error is

$\boldsymbol{E}_{\left.\nabla^{2} p(\boldsymbol{x})\right|_{x=x_{I}}}=\boldsymbol{I}^{\mathrm{T}} \boldsymbol{E}_{\left.\nabla^{(2 s)} p(\boldsymbol{x})\right|_{x=x_{I}}}$

In practices, Eq. (24) can be applied to discretise the Poisson's equation at all particle positions and/or to directly approximate $\nabla^{2} p(\boldsymbol{x})$ at a point $\boldsymbol{x}_{I}$ coinciding with a particle location, where $p_{I}$ is known. However, to estimate $\nabla^{2} p(\boldsymbol{x})$ at a point that does not coincide with any particles, $p_{I}$ needs to be numerically interpolated using $p_{j}$. To do so, estimation of $\left.\nabla^{(2 c)} p(\boldsymbol{x})\right|_{\boldsymbol{x}=\boldsymbol{x}_{I}}$ in Eq. (1) is required and achieved by substituting Eqs. (20)-(23) to Eq. (15),

$$
\begin{array}{r}
\left\langle\left.\nabla^{(2 c)} p(\boldsymbol{x})\right|_{\boldsymbol{x}=\boldsymbol{x}_{I}}\right\rangle=\boldsymbol{M}_{2 c, I}^{-1} \sum_{j=1}^{N} \frac{w_{j I}}{d_{j I}^{4}} \boldsymbol{r}_{j I}^{(2 c)}\left(p_{j}-p_{I}\right)-\boldsymbol{M}_{2 c, I}^{-1} \sum_{j=1}^{N} \frac{w_{j I}}{d_{j I}^{4}} \boldsymbol{r}_{j I}^{(2 c)} \boldsymbol{r}_{j I}^{\mathrm{T}} \\
\left\langle\left.\nabla p(\boldsymbol{x})\right|_{\boldsymbol{x}=\boldsymbol{x}_{I}}\right\rangle-\frac{1}{2} \boldsymbol{M}_{2 c, I}^{-1} \sum_{j=1}^{N} \frac{w_{j I}}{d_{j I}^{4}} \boldsymbol{r}_{j I}^{(2 c)}\left(\boldsymbol{r}_{j I}^{(2 s)}\right)^{\mathrm{T}}\left\langle\left.\nabla^{(2 s)} p(\boldsymbol{x})\right|_{\boldsymbol{x}=\boldsymbol{x}_{I}}\right\rangle
\end{array}
$$

with a leading truncation error of

$$
\begin{aligned}
\boldsymbol{E}_{\left.\nabla^{(2 c)} p(\boldsymbol{x})\right|_{x=x_{I}}=} & -\boldsymbol{M}_{2 c, I}^{-1} \sum_{j=1}^{N} \frac{w_{j I}}{d_{j I}^{4}} \boldsymbol{r}_{j I}^{(2 c)} \boldsymbol{r}_{j I}^{\mathrm{T}} \boldsymbol{E}_{\left.\nabla p(\boldsymbol{x})\right|_{x=x_{I}}}-\frac{1}{2} \boldsymbol{M}_{2 c, I}^{-1} \sum_{j=1}^{N} \frac{w_{j I}}{d_{j I}^{4}} \boldsymbol{r}_{j I}^{(2 c)} \\
& \left(\boldsymbol{r}_{j I}^{(2 s)}\right)^{\mathrm{T}} \boldsymbol{E}_{\left.\nabla^{(2 s)} p(\boldsymbol{x})\right|_{x=x_{I}}}-\left.\frac{1}{6} \boldsymbol{M}_{2 c, I}^{-1} \sum_{j=1}^{N} \frac{w_{j I}}{d_{j I}^{4}} \boldsymbol{r}_{j I}^{(2 c)}\left(\boldsymbol{r}_{j I}^{(3)}\right)^{\mathrm{T}} \nabla^{(3)} p(\boldsymbol{x})\right|_{\boldsymbol{x}=\boldsymbol{x}_{I}}
\end{aligned}
$$

For convenience of deriving the interpolation function, Eqs. (20), (22) and (26) are, respectively, rewritten in a summation form, i.e.

$$
\begin{aligned}
& \left\langle\left.\nabla p(\boldsymbol{x})\right|_{\boldsymbol{x}=\boldsymbol{x}_{I}}\right\rangle=\sum_{j=1}^{N} \boldsymbol{\Phi}_{j I}^{g}\left(p_{j}-p_{I}\right) \\
& \left\langle\left.\nabla^{(2 s)} p(\boldsymbol{x})\right|_{\boldsymbol{x}=\boldsymbol{x}_{I}}\right\rangle=\sum_{j=1}^{N} \boldsymbol{\Phi}_{j I}^{s}\left(p_{j}-p_{I}\right) \\
& \left\langle\left.\nabla^{(2 c)} p(\boldsymbol{x})\right|_{\boldsymbol{x}=\boldsymbol{x}_{I}}\right\rangle=\sum_{j=1}^{N} \boldsymbol{\Phi}_{j I}^{c}\left(p_{j}-p_{I}\right)
\end{aligned}
$$

where

$$
\begin{aligned}
& \boldsymbol{\Phi}_{j I}^{g}=\boldsymbol{M}_{1 q, I}^{-1}\left(\frac{w_{j I}}{d_{j I}^{2}} \boldsymbol{q}_{j I}-\sum_{k=1}^{N} \frac{w_{k I}}{d_{k I}^{2}} \boldsymbol{q}_{k I}\left(\boldsymbol{r}_{k I}^{(2 c)}\right)^{\mathrm{T}} \frac{w_{j I}}{d_{j I}^{4}} \boldsymbol{r}_{j I}^{(2 c)}-\sum_{k=1}^{N} \frac{w_{k I}}{d_{k I}^{2}} \boldsymbol{q}_{k I} \boldsymbol{\Pi}_{k I}^{\mathrm{T}} \boldsymbol{M}_{2 s, I}^{-1} \boldsymbol{\Gamma}_{j I}\right) \\
& \boldsymbol{\Phi}_{j I}^{s}=2 \boldsymbol{M}_{2 s, I}^{-1}\left(\boldsymbol{\Gamma}_{j I}-\sum_{k=1}^{N} \frac{w_{k I}}{d_{k I}^{4}} \boldsymbol{\Pi}_{k I} \boldsymbol{G}_{k I}^{\mathrm{T}} \boldsymbol{\Phi}_{k I}^{g}\right) \text { and } \\
& \boldsymbol{\Phi}_{j I}^{c}=\boldsymbol{M}_{2 c, I}^{-1}\left(\frac{w_{j I}}{d_{j I}^{4}} \boldsymbol{r}_{j I}^{(2 c)}-\sum_{k=1}^{N} \frac{w_{k I}}{d_{k I}^{4}} \boldsymbol{r}_{k I}^{(2 c)} \boldsymbol{r}_{k I}^{\mathrm{T}} \boldsymbol{\Phi}_{j I}^{g}-\frac{1}{2} \sum_{k=1}^{N} \frac{w_{k I}}{d_{k I}^{4}} \boldsymbol{r}_{k I}^{(2 c)}\left(\boldsymbol{r}_{k I}^{(2 s)}\right)^{\mathrm{T}} \boldsymbol{\Phi}_{j I}^{s}\right) .
\end{aligned}
$$

Consequently, Eq. (24) can be re-written as

$$
\left\langle\left.\nabla^{2} p(\boldsymbol{x})\right|_{\boldsymbol{x}=\boldsymbol{x}_{I}}\right\rangle=\boldsymbol{I}^{\mathrm{T}} \sum_{j=1}^{N} \boldsymbol{\Phi}_{j I}^{s}\left(p_{j}-p_{I}\right)
$$

Multiplying Eq. (1) by $w_{j I} / d_{j l}$, taking the sum of resultant equations at all particles in $\Omega_{I}$, it leads to

$$
\begin{aligned}
& \sum_{j=1}^{N} \frac{w_{j I}\left(p_{j}-p_{I}\right)}{d_{j I}} \approx \sum_{j=1}^{N} \frac{w_{j I}}{d_{j I}} \\
& {\left[\boldsymbol{r}_{j I}^{\mathrm{T}} \nabla p(\boldsymbol{x})+\frac{1}{2}\left(\boldsymbol{r}_{j I}^{(2 s)}\right)^{\mathrm{T}} \nabla^{(2 s)} p(\boldsymbol{x})+\left(\boldsymbol{r}_{j I}^{(2 c)}\right)^{\mathrm{T}} \nabla^{(2 c)} p(\boldsymbol{x})+\frac{1}{6}\left(\boldsymbol{r}_{j I}^{(3)}\right)^{\mathrm{T}} \nabla^{(3)} p(\boldsymbol{x})\right]_{\boldsymbol{x}=\boldsymbol{x}_{I}}}
\end{aligned}
$$

Substituting Eqs. (28)-(30) into Eq. (32), the expression for interpolating $p_{I}$ and its truncation error $E_{p}$ can be formulated as

$$
\left\langle p_{I}\right\rangle=\frac{1}{M_{0}} \sum_{j=1}^{N} \Phi_{j I} p_{j}
$$

$$
\begin{aligned}
& E_{p}=-\left.\frac{1}{6 M_{0}} \sum_{j=1}^{N} \frac{w_{j I}}{d_{j I}}\left(\boldsymbol{r}_{j I}^{(3)}\right)^{\mathrm{T}} \nabla^{(3)} p(\boldsymbol{x})\right|_{\boldsymbol{x}=\boldsymbol{x}_{I}} \\
& -\frac{1}{M_{0}} \sum_{j=1}^{N} \frac{w_{j I}}{d_{j I}}\left(\boldsymbol{r}_{j I}^{\mathrm{T}} \boldsymbol{E}_{\left.\nabla p(\boldsymbol{x})\right|_{x=x_{I}}}+\frac{1}{2}\left(\boldsymbol{r}_{j I}^{(2 s)}\right)^{\mathrm{T}} \boldsymbol{E}_{\nabla}\left(\left.2 s p(\boldsymbol{x})\right|_{x=x_{I}}+\left(\boldsymbol{r}_{j I}^{(2 c)}\right)^{\mathrm{T}} \boldsymbol{E}_{\nabla}{ }_{\nabla}^{\left.(2 c) p(\boldsymbol{x})\right|_{x=x_{I}}}\right)\right.
\end{aligned}
$$

where

$$
\begin{aligned}
M_{0} & =\sum_{j=1}^{N} \frac{w_{j I}}{d_{j I}}\left(1-\boldsymbol{r}_{j I}^{\mathrm{T}} \sum_{k=1}^{N} \boldsymbol{\Phi}_{k I}^{g}-\frac{1}{2}\left(\boldsymbol{r}_{j I}^{(2 s)}\right)^{\mathrm{T}} \sum_{k=1}^{N} \boldsymbol{\Phi}_{k I}^{s}-\left(\boldsymbol{r}_{j I}^{(2 c)}\right)^{\mathrm{T}} \sum_{k=1}^{N} \boldsymbol{\Phi}_{k I}^{c}\right), \\
\Phi_{j I} & =\frac{w_{j I}}{d_{j I}}\left(1-\boldsymbol{r}_{j I}^{\mathrm{T}} \sum_{k=1}^{N} \boldsymbol{\Phi}_{k I}^{g}-\frac{1}{2}\left(\boldsymbol{r}_{j I}^{(2 s)}\right)^{\mathrm{T}} \sum_{k=1}^{N} \boldsymbol{\Phi}_{k I}^{s}-\left(\boldsymbol{r}_{j I}^{(2 c)}\right)^{\mathrm{T}} \sum_{k=1}^{N} \boldsymbol{\Phi}_{k I}^{c}\right) .
\end{aligned}
$$

By replacing $p_{I}$ in Eq. (10) or Eq. (31), the Laplacian at a point that does not coincide with any particles can be obtained, i.e.

$$
\left\langle\left.\nabla^{2} p(\boldsymbol{x})\right|_{\boldsymbol{x}=\boldsymbol{x}_{I}}\right\rangle=\boldsymbol{I}^{\mathrm{T}} \sum_{j=1}^{N} \boldsymbol{\Phi}_{j I}^{s}\left(p_{j}-\left\langle p_{I}\right\rangle\right)
$$

As shown above, the leading truncation errors for numerical interpolation, gradient estimation and Laplacian discretisation in the QSFDI are proportional to the third derivatives $\left.\nabla^{(3)} p(\boldsymbol{x})\right|_{\boldsymbol{x}=\boldsymbol{x}_{I}}$, suggesting that the QSFDI provides exact solutions for quadric polynomials. 


\section{Appendix 2: Error Analysis of CSPM and improvement}

The CSPM is proposed by Chen et al. (1999), which is derived based on the kernel integration of the conventional Taylor's expansion

$$
\begin{aligned}
p_{j}-p_{I}= & \left.\boldsymbol{r}_{j I}^{\mathrm{T}} \nabla p(\boldsymbol{x})\right|_{\boldsymbol{x}=\boldsymbol{x}_{I}}+\left.\frac{1}{2}\left(\boldsymbol{r}_{j I}^{(2)}\right)^{\mathrm{T}} \nabla^{(2)} p(\boldsymbol{x})\right|_{\boldsymbol{x}=\boldsymbol{x}_{I}} \\
& +\left.\frac{1}{6}\left(\boldsymbol{r}_{j I}^{(3)}\right)^{\mathrm{T}} \nabla^{(3)} p(\boldsymbol{x})\right|_{\boldsymbol{x}=\boldsymbol{x}_{I}}+\ldots
\end{aligned}
$$

in which $\left(\boldsymbol{r}_{j I}^{\mathrm{T}} \nabla\right){ }^{2} p(\boldsymbol{x})$ and $\left.\left(\boldsymbol{r}_{j I}^{\mathrm{T}} \nabla\right){ }^{3} p(\boldsymbol{x})\right|_{\boldsymbol{x}=\boldsymbol{x}_{I}}$ are rewritten as $\left.\left(\boldsymbol{r}_{j I}^{(2)}\right)^{\mathrm{T}} \nabla^{(2)} p(\boldsymbol{x})\right|_{\boldsymbol{x}=\boldsymbol{x}_{I}}$ and $\left.\left(\boldsymbol{r}_{j I}^{(3)}\right)^{\mathrm{T}} \nabla^{(3)} p(\boldsymbol{x})\right|_{\boldsymbol{x}=\boldsymbol{x}_{I}}$, respectively, and

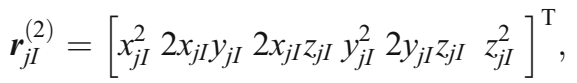

$\nabla^{(2)}=\left[\begin{array}{llllll}\frac{\partial^{2}}{\partial x^{2}} & \frac{\partial^{2}}{\partial x \partial y} & \frac{\partial^{2}}{\partial x \partial z} & \frac{\partial^{2}}{\partial y^{2}} & \frac{\partial^{2}}{\partial y \partial z} & \frac{\partial^{2}}{\partial z^{2}}\end{array}\right]^{\mathrm{T}}$.

Multiplying Eq. (36) by $\nabla^{(2)} W_{j I}$, where $W_{j I}$ is the kernel function, and integrating over the support domain $\Omega_{I}$, yields,

$$
\begin{aligned}
& \sum_{j=1}^{N} \frac{\nabla^{(2)} W_{j l} m_{j}}{\rho_{j}}\left(p_{j}-p_{I}\right)=\left.\sum_{j=1}^{N} \frac{\nabla^{(2)} W_{j l} m_{j}}{\rho_{j}} \boldsymbol{r}_{j I}^{\mathrm{T}} \nabla p(\boldsymbol{x})\right|_{x=x_{I}} \\
& +\left.\frac{1}{2} \sum_{j=1}^{N} \frac{\nabla^{(2)} W_{j l} m_{j}}{\rho_{j}}\left(r_{j I}^{(2)}\right)^{\mathrm{T}} \nabla^{(2)} p(\boldsymbol{x})\right|_{x=x_{I}}+\left.\frac{1}{6} \sum_{j=1}^{N} \frac{\nabla^{(2)} W_{j l} m_{j}}{\rho_{j}}\left(r_{j I}^{(3)}\right)^{\mathrm{T}} \nabla^{(3)} p(\boldsymbol{x})\right|_{x=x_{j}}+\ldots
\end{aligned}
$$

where the kernel integration has been written in a summation from Chen et al. (1999) and $m_{j} / \rho_{j}$ is the volume (area) represented by particle $j$. By ignoring the last two terms in the righthand side of Eq. (37), the 2nd derivative term $\left.\nabla^{(2)} p(\boldsymbol{x})\right|_{\boldsymbol{x}=\boldsymbol{x}_{I}}$ can be approximated by using

$\left\langle\left.\nabla^{(2)} p(\boldsymbol{x})\right|_{x=x_{i}}\right\rangle=2 \boldsymbol{M}_{2, \mathrm{CSPM}}^{-1}\left(\sum_{j=1}^{N} \frac{\nabla^{(2)} W_{j l} m_{j}}{\rho_{j}}\left(p_{j}-p_{I}\right)-\left.\sum_{j=1}^{N} \frac{\nabla^{(2)} W_{j j} m_{j}}{\rho_{j}} \boldsymbol{r}_{j I}^{\mathrm{T}} \nabla p(\boldsymbol{x})\right|_{x=x_{I}}\right)$

where $\boldsymbol{M}_{2, \mathrm{CSPM}}=\sum_{j=1}^{N} \nabla^{(2)} W_{j I} \frac{m_{j}}{\rho_{j}\left(\boldsymbol{r}_{j I}^{(2)}\right)}$, is a matrix with size of $6 \times 6$ for 3 D problems or $3 \times 3$ for 2 D problems. In numerical practices, $\left.\nabla p(\boldsymbol{x})\right|_{\boldsymbol{x}=\boldsymbol{x}_{I}}$ is often unavailable and therefore needs to be numerically estimated. To do so, one may multiply Eq. (36) by $\nabla W_{j I}$ and integrate the resultant equation over $\Omega_{I}$

$$
\begin{aligned}
\sum_{j=1}^{N} \frac{\nabla W_{j I} m_{j}}{\rho_{j}}\left(p_{j}-p_{I}\right)=\left.\sum_{j=1}^{N} \frac{\nabla W_{j I} m_{j}}{\rho_{j}} r_{j I}^{\mathrm{T}} \nabla p(\boldsymbol{x})\right|_{\boldsymbol{x}=\boldsymbol{x}_{I}} \\
+\left.\frac{1}{2} \sum_{j=1}^{N} \frac{\nabla W_{j I} m_{j}}{\rho_{j}}\left(\boldsymbol{r}_{j I}^{(2)}\right)^{\mathrm{T}} \nabla^{(2)} p(\boldsymbol{x})\right|_{\boldsymbol{x}=\boldsymbol{x}_{I}} \\
\quad+\left.\frac{1}{6} \sum_{j=1}^{N} \frac{\nabla W_{j I} m_{j}}{\rho_{j}}\left(\boldsymbol{r}_{j I}^{(3)}\right)^{\mathrm{T}} \nabla^{(3)} p(\boldsymbol{x})\right|_{\boldsymbol{x}=\boldsymbol{x}_{I}}+\ldots
\end{aligned}
$$

Chen et al. (1999) ignored the last three terms in the righthand side of Eq. (39), yielding the scheme for gradient estimation

$$
\left\langle\left.\nabla p(\boldsymbol{x})\right|_{\boldsymbol{x}=\boldsymbol{x}_{I}}\right\rangle=\boldsymbol{M}_{1, \mathrm{CSPM}}^{-1} \sum_{j=1}^{N} \frac{\nabla W_{j I} m_{j}}{\rho_{j}}\left(p_{j}-p_{I}\right)
$$

in which $\boldsymbol{M}_{1, \mathrm{CSPM}}=\sum_{j=1}^{N} \nabla W_{j I} \frac{m_{j}}{\rho_{j} \boldsymbol{r}_{j l}^{\mathrm{T}}}$, is a $3 \times 3$ matrix for $3 \mathrm{D}$ problems or $2 \times 2$ matrix for $2 \mathrm{D}$ problems. The truncation error of Eq. (40) can be expressed by

$$
-\frac{1}{2} \boldsymbol{M}_{1, \mathrm{CSPM}}^{-1}\left\{\left.\sum_{j=1}^{N} \frac{\nabla W_{j} m_{j}}{\rho_{j}}\left(\boldsymbol{r}_{j l}^{(2)}\right)^{\mathrm{T}} \nabla^{(2)} p(\boldsymbol{x})\right|_{x=x_{j}}+\frac{1}{3} \sum_{j=1}^{N} \frac{\nabla W_{j} m_{j}}{\rho_{j}}\left(\boldsymbol{r}_{j l}^{(3)}\right)^{\mathrm{T}} \nabla^{(3)} p\left(\left.\boldsymbol{x}\right|_{x=x_{l}}\right\}\right.
$$

Replacing $\nabla p(\boldsymbol{x})$ in Eq. (38) by $\left\langle\left.\nabla p(\boldsymbol{x})\right|_{\boldsymbol{x}=\boldsymbol{x}_{I}}\right\rangle$ specified by Eq. (40), the following equation in the CSPM to discretise the Laplacian can be achieved

$$
\begin{aligned}
& \left\langle\left.\nabla^{2} p(\boldsymbol{x})\right|_{x=x_{I}}\right\rangle=2 \boldsymbol{I}_{\mathrm{CSPM}}^{\mathrm{T}} \boldsymbol{M}_{2, \mathrm{CSPM}}^{-1} \\
& \left(\sum_{j=1}^{N} \frac{\nabla^{(2)} W_{j l} m_{j}}{\rho_{j}}\left(p_{j}-p_{I}\right)-\sum_{j=1}^{N} \frac{\nabla^{(2)} W_{j l} m_{j}}{\rho_{j}} \boldsymbol{r}_{j l}^{\mathrm{T}} \boldsymbol{M}_{1, \mathrm{CSPM}}^{-1} \sum_{k=1}^{N} \frac{\nabla W_{k l} m_{k}}{\rho_{k}}\left(p_{k}-p_{I}\right)\right)
\end{aligned}
$$

where $I_{\mathrm{CSPM}}=\left[\begin{array}{llllll}1 & 0 & 0 & 1 & 0 & 1\end{array}\right]^{\mathrm{T}}$. The truncation error of Eq. (41) is

$$
\begin{aligned}
\boldsymbol{E}_{\mathrm{CSPM}}=\boldsymbol{I}_{\mathrm{CSPM}}^{\mathrm{T}} \boldsymbol{M}_{2, \mathrm{CPPM}}^{-1} \sum_{j=1}^{N} \frac{\nabla^{(2)} W_{j l} m_{j}}{\rho_{j}} \boldsymbol{r}_{j l}^{\mathrm{T}} & \\
\left(\left.\boldsymbol{M}_{1, \mathrm{CSPM}}^{-1} \sum_{k=1}^{N} \frac{\nabla W_{k l} m_{k}}{\rho_{k}}\left(\boldsymbol{r}_{k l}^{(2)}\right)^{\mathrm{T}} \nabla^{(2)} p(\boldsymbol{x})\right|_{x=x_{i}}\right. & \left.+\frac{1}{3} \boldsymbol{M}_{1, \mathrm{CSPM}}^{-1} \sum_{k=1}^{N} \frac{\nabla W_{k l} m_{k}}{\rho_{k}}\left(\boldsymbol{r}_{k l}^{(3)}\right)^{\mathrm{T}} \nabla^{(3)} p(\boldsymbol{x})_{x=x_{i}}\right) \\
& -\left.\frac{1}{3} \boldsymbol{I}_{\mathrm{CSPM}}^{\mathrm{T}} \boldsymbol{M}_{2, \mathrm{CSPM}}^{-1} \sum_{j=1}^{N} \frac{\nabla^{(2)} W_{j l} m_{j}}{\rho_{j}}\left(\boldsymbol{r}_{j l}^{(3)}\right)^{\mathrm{T}} \nabla^{(3)} p(\boldsymbol{x})\right|_{x=x_{i}}
\end{aligned}
$$

In the cases with a regular particle distribution and a uniform spacing, $\sum_{j=1}^{N} \nabla^{(2)} W_{j I} \frac{m_{j}}{\rho_{j} \boldsymbol{r}_{j I}^{\top}=0}$ and $\sum_{j=1}^{N} \nabla^{(2)} W_{j I} \frac{m_{j}}{\rho_{j}\left(\boldsymbol{r}_{j I}^{(3)}\right)^{\mathrm{T}}=0}$, consequently, the leading truncation error of Eq. (41) is in the order of $O\left(\left.\boldsymbol{I}_{\mathrm{CSPM}}^{\mathrm{T}} \boldsymbol{M}_{2, \mathrm{CSPM}}^{-1}\left(\boldsymbol{r}_{j I}^{\mathrm{T}} \nabla\right){ }^{4} p(\boldsymbol{x})\right|_{\boldsymbol{x}=\boldsymbol{x}_{I}}\right)$, if the kernel is symmetrical about $\boldsymbol{x}_{I}$ and the integration domain is full. This is consistent with the conclusion by Chen et al. (1999). Nevertheless, if the particles are randomly distributed, the leading truncation error of Eq. (41) becomes 
$\boldsymbol{E}_{\mathrm{CSPM}}=\left.\boldsymbol{I}_{\mathrm{CSPM}}^{\mathrm{T}} \boldsymbol{M}_{2, \mathrm{CSPM}}^{-1} \sum_{j=1}^{N} \frac{\nabla^{(2)} W_{j l} m_{j}}{\rho_{j}} \boldsymbol{r}_{j l}^{\mathrm{T}} \boldsymbol{M}_{1, \mathrm{CSPM}}^{-1} \sum_{k=1}^{N} \frac{\nabla W_{k l} m_{k}}{\rho_{k}}\left(\boldsymbol{r}_{k I}^{(2)}\right)^{\mathrm{T}} \nabla^{(2)} p(\boldsymbol{x})\right|_{\boldsymbol{x}=\boldsymbol{x}_{I}}$

The schemes developed by Schwaiger (2008) are also based on the kernel integration of Eq. (36). Its distinguishing feature is that $\left(\boldsymbol{r}_{j I}^{(2)}\right)^{\mathrm{T}} \nabla^{(2)} p(\boldsymbol{x})$ in Eq. (36) is replaced by $\left(\boldsymbol{r}_{j I}^{(2 s)}\right)^{\mathrm{T}} \boldsymbol{\nabla}^{(2 s)} p(\boldsymbol{x})$, assuming that the kernel integration of $\left(\boldsymbol{r}_{j I}^{(2 c)}\right)^{\mathrm{T}} \boldsymbol{\nabla}^{(2 c)} p(\boldsymbol{x})$ is zero, i.e.

$p_{j}-p_{I}=\left.\left(\boldsymbol{r}_{j I}^{\mathrm{T}} \nabla\right) p(\boldsymbol{x})\right|_{x=\boldsymbol{x}_{I}}+\left.\frac{1}{2}\left(\boldsymbol{r}_{j I}^{(2 s)}\right)^{\mathrm{T}} \nabla^{(2 s)} p(\boldsymbol{x})\right|_{x=x_{I}}+\left.\frac{1}{6}\left(\boldsymbol{r}_{j I}^{(3)}\right)^{\mathrm{T}} \nabla^{(3)} p(\boldsymbol{x})\right|_{\boldsymbol{x}=\boldsymbol{x}_{I}}+\ldots$ $\begin{array}{lll}\text { Multiplying } \quad \text { Eq. } & \text { (44) by }\end{array}$ $\nabla^{\check{ }} W_{j I}=\frac{1}{d_{j I}^{2}}\left[x_{j I} \frac{\partial W_{j I}}{\partial x} \quad y_{j I} \frac{\partial W_{j I}}{\partial y} \quad z_{j I} \frac{\partial W_{j I}}{\partial z}\right]^{\mathrm{T}}$ and integrating over the domain $\Omega$, it leads to

$\left\langle\left.\nabla^{2} p(\boldsymbol{x})\right|_{x=x_{I}}\right\rangle=2 \boldsymbol{I}^{\mathrm{T}} \boldsymbol{M}_{2, \mathrm{CSPH}}^{-1}\left(\sum_{j=1}^{N} \frac{\nabla^{\nu} W_{j l} m_{j}}{\rho_{j}}\left(p_{j}-p_{I}\right)-\sum_{j=1}^{N} \frac{\nabla^{\nu} W_{j I} m_{j}}{\rho_{j}} \boldsymbol{r}_{j I}^{\mathrm{T}}\left\langle\left.\nabla p(\boldsymbol{x})\right|_{\boldsymbol{x}=\boldsymbol{x}_{I}}\right\rangle\right)$

where $\boldsymbol{M}_{2, \mathrm{CSPH}}=\sum_{j=1}^{N} \nabla^{\swarrow} W_{j I} \frac{m_{j}}{\rho_{j}\left(\boldsymbol{r}_{j I}^{(2 s)}\right)^{\mathrm{T}}}$ is a matrix with size of 3 $\times 3$ for $3 \mathrm{D}$ problems or $2 \times 2$ for $2 \mathrm{D}$ problems. $\left\langle\left.\nabla p(\boldsymbol{x})\right|_{\boldsymbol{x}=\boldsymbol{x}_{I}}\right\rangle$ in Eq. (45) is also estimated using Eq. (40). Therefore, the truncation error of Eq. (45) is

$$
\begin{aligned}
& \boldsymbol{E}_{\mathrm{CSPH}}=\boldsymbol{I}^{\mathrm{T}} \boldsymbol{M}_{2, \mathrm{CSPH}}^{-1} \sum_{j=1}^{N} \frac{\nabla^{\check{ }} W_{j I} m_{j}}{\rho_{j}} \boldsymbol{r}_{j I}^{\mathrm{T}}\left(\left.\frac{1}{2} \boldsymbol{M}_{1, \mathrm{CSPM}}^{-1} \sum_{k=1}^{N} \frac{\nabla W_{k I} m_{k}}{\rho_{k}}\left(\boldsymbol{r}_{k I}^{(2)}\right)^{\mathrm{T}} \nabla^{(\mathbf{2})} p(\boldsymbol{x})\right|_{\boldsymbol{x}=\boldsymbol{x}_{I}}+\left.\frac{1}{3} \boldsymbol{M}_{1}^{-1} \sum_{k=1}^{N} \frac{\nabla W_{k I} m_{k}}{\rho_{k}}\left(\boldsymbol{r}_{k I}^{(3)}\right)^{\mathrm{T}} \nabla^{(3)} p(\boldsymbol{x})\right|_{\boldsymbol{x}=\boldsymbol{x}_{I}}\right) \\
& -\left.\boldsymbol{I}^{\mathrm{T}} \boldsymbol{M}_{2, \mathrm{CSPH}}^{-1} \sum_{j=1}^{N} \frac{\nabla^{\check{ } W_{j I} m_{j}}}{\rho_{j}}\left(\boldsymbol{r}_{j I}^{(2 c)}\right)^{\mathrm{T}} \nabla^{(\mathbf{2 c})} p(\boldsymbol{x})\right|_{\boldsymbol{x}=\boldsymbol{x}_{I}}-\left.\frac{1}{3} \boldsymbol{I}^{\mathrm{T}} \boldsymbol{M}_{2, \mathrm{CSPH}}^{-1} \sum_{j=1}^{N} \frac{\nabla^{\check{ }} W_{j I} m_{j}}{\rho_{j}}\left(\boldsymbol{r}_{j I}^{(3)}\right)^{\mathrm{T}} \nabla^{(3)} p(\boldsymbol{x})\right|_{\boldsymbol{x}=\boldsymbol{x}_{I}}+\ldots
\end{aligned}
$$

The leading truncation error of Schwaiger's scheme is sourced from (1) the leading truncation error of the gradient estimation using Eq. (40) and (2) ignoring the crossderivative terms, $\left(\boldsymbol{r}_{j I}^{(2 c)}\right)^{\mathrm{T}} \nabla^{(2 c)} p(\boldsymbol{x})$, in Eq. (44). However, only the leading truncation sourced from (1) is shown in the CSPM (Eq. (41)). Such terms can be eliminated if a higher-order gradient estimation scheme is introduced. For example, truncating the last two terms on the right-hand side of Eq. (37), it leads to

$$
\left\langle\nabla p(\boldsymbol{x})_{\boldsymbol{x}=\boldsymbol{x}_{l}}\right\rangle=\boldsymbol{M}_{1, \mathrm{CSPM}}^{-1} \sum_{j=1}^{N} \frac{\nabla W_{j I} m_{j}}{\rho_{j}}\left(p_{j}-p_{I}\right)-\left.\frac{1}{2} \boldsymbol{M}_{1, \mathrm{CSPM}}^{-1} \sum_{j=1}^{N} \frac{\nabla W_{j l} m_{j}}{\rho_{j}}\left(\boldsymbol{r}_{j I}^{(2)}\right)^{\mathrm{T}} \nabla^{(2)} p(\boldsymbol{x})\right|_{x=x_{I}}
$$

with a leading truncation error of

$$
-\left.\frac{1}{6} \boldsymbol{M}_{1, \mathrm{CSPM}}^{-1} \sum_{j=1}^{N} \frac{\nabla W_{j I} m_{j}}{\rho_{j}}\left(\boldsymbol{r}_{j I}^{(3)}\right)^{\mathrm{T}} \nabla^{(3)} p(\boldsymbol{x})\right|_{\boldsymbol{x}=\boldsymbol{x}_{I}}
$$

Substituting Eq. (47) into Eq. (38), and multiplying the resultant equation by $\boldsymbol{I}_{\mathrm{CSPM}}^{\mathrm{T}}$, it leads to the formula to discretise the Laplacian,

$$
\begin{aligned}
& \left\langle\left.\nabla^{2} p(\boldsymbol{x})\right|_{\boldsymbol{x}=\boldsymbol{x}_{I}}\right\rangle=2 \boldsymbol{I}_{\mathrm{CSPM}}^{\mathrm{T}} \boldsymbol{M}_{2, \mathrm{ICSPM}}^{-1} \\
& \quad\left\{\sum_{j=1}^{N} \frac{\nabla^{(2)} W_{j I} m_{j}}{\rho_{j}}\left(p_{j}-p_{I}\right)-\sum_{j=1}^{N} \frac{\nabla^{(2)} W_{j I} m_{j}}{\rho_{j}} \boldsymbol{r}_{j I}^{\mathrm{T}} \boldsymbol{M}_{1, \mathrm{CSPM}}^{-1} \sum_{k=1}^{N} \frac{\nabla W_{j I} m_{k}}{\rho_{k}}\left(p_{k}-p_{I}\right)\right\}
\end{aligned}
$$

in which

$$
\boldsymbol{M}_{2, \mathrm{ICSPM}}=\boldsymbol{M}_{2, \mathrm{CSPM}}-\sum_{j=1}^{N}\left(\frac{\nabla^{(2)} W_{j I} m_{j}}{\rho_{j}} \boldsymbol{r}_{j I}^{\mathrm{T}} \boldsymbol{M}_{1, \mathrm{CSPM}}^{-1} \sum_{k=1}^{N} \frac{\nabla W_{k I} m_{k}}{\rho_{k}}\left(\boldsymbol{r}_{k I}^{(2)}\right)^{\mathrm{T}}\right)
$$

The leading truncation error of Eq. (48) for randomly distributed particles is

$E_{\mathrm{ICSPM}}=\frac{1}{3} \boldsymbol{I}_{\mathrm{CSPM}}^{\mathrm{T}} \boldsymbol{M}_{2, \mathrm{CSPM}}^{-1}\left\{\sum_{j=1}^{N} \frac{\nabla^{(2)} W_{j I} m_{j}}{\rho_{j}} \boldsymbol{r}_{j I}^{\mathrm{T}} \boldsymbol{M}_{1, C S P M}^{-1} \sum_{k=1}^{N} \frac{\nabla W_{k l} m_{k}}{\rho_{k}}\left(\boldsymbol{r}_{k I}^{(3)}\right)^{\mathrm{T}} \nabla^{(3)} p(\boldsymbol{x})\left|x=\boldsymbol{x}_{l}-\sum_{j=1}^{N} \frac{\nabla^{(2)} W_{j I} m_{j}}{\rho_{j}}\left(\boldsymbol{r}_{k I}^{(3)}\right)^{\mathrm{T}} \nabla^{(3)} p(\boldsymbol{x})\right|_{\boldsymbol{x}=\boldsymbol{x}_{l}}\right\}$ 
Open Access This article is licensed under a Creative Commons Attribution 4.0 International License, which permits use, sharing, adaptation, distribution and reproduction in any medium or format, as long as you give appropriate credit to the original author(s) and the source, provide a link to the Creative Commons licence, and indicate if changes were made. The images or other third party material in this article are included in the article's Creative Commons licence, unless indicated otherwise in a credit line to the material. If material is not included in the article's Creative Commons licence and your intended use is not permitted by statutory regulation or exceeds the permitted use, you will need to obtain permission directly from the copyright holder. To view a copy of this licence, visit http://creativecommons.org/licenses/by/4.0/.

\section{References}

Abbaszadeh M, Dehghan M (2019) The interpolating element-free Galerkin method for solving Korteweg-de Vries-Rosenauregularized long-wave equation with error analysis. Nonlinear Dyn 96(2):1345-1365. https://doi.org/10.1007/S11071-019-04858-1

Brookshaw L (1985) A method of calculating radiative heat diffusion in particle simulations. Publ Astron Soc Aust 6(2):207-210. https:// doi.org/10.1017/S1323358000018117

Chen JK, Beraun JE, Carney TC (1999) A corrective smoothed particle method for boundary value problems in heat conduction. Int $\mathrm{J}$ Numer Methods Eng 46(2):231-252. https://doi.org/10.1002/ (SICI)1097-020719990920)46:2<231::AID-NME672>3.0.CO;2-K

Cummins SJ, Rudman M (1999) An SPH projection method. J Comput Phys 152(2):584-607. https://doi.org/10.1006/jcph.1999.6246

Dehghan M, Abbaszadeh M (2018) Variational multiscale element-free Galerkin method combined with the moving Kriging interpolation for solving some partial differential equations with discontinuous solutions. Comput Appl Math 37(3):3869-3905. https://doi.org/10. 1007/s40314-017-0546-6

Dehghan M, Abbaszadeh M (2019) Error analysis and numerical simulation of magnetohydrodynamics (MHD) equation based on the interpolating element free Galerkin (IEFG) method. Appl Numer Math 137:252-273. https://doi.org/10.1016/j.apnum.2018.10.004

Fatehi R, Manzari MT (2011) Error estimation in smoothed particle hydrodynamics and a new scheme for second derivatives. Comput Math Appl 61(2):482-498. https://doi.org/10.1016/j.camwa.2010.11.028

Gotoh H, Khayyer A (2016) Current achievements and future perspectives for projection-based particle methods with applications in ocean engineering. J Ocean Eng Marine Energy 2(3):251-278. https://doi.org/10.1007/s40722-016-0049-3

Gotoh H, Khayyer A, Ikari H, Arikawa T, Shimosako K (2014) On enhancement of incompressible SPH method for simulation of violent sloshing flows. Appl Ocean Res 46:104-115. https://doi.org/10. 1016/j.apor.2014.02.005

Hu XY, Adams NA (2007) An incompressible multi-phase SPH method. J Comput Phys 227(1):264-278. https://doi.org/10.1016/j.jcp.2007.07.013

Ikari H, Khayyer A, Gotoh H (2015) Corrected higher order Laplacian for enhancement of pressure calculation by projection-based particle methods with applications in ocean engineering. J Ocean Eng Marine Energy 1(4):361-376. https://doi.org/10.1007/s40722-015-0026-2

Khayyer A, Gotoh H (2010) A higher order Laplacian model for enhancement and stabilization of pressure calculation by the MPS method. Appl Ocean Res 32(1):124-131. https://doi.org/10.1016/j.apor. 2010.01.001
Khayyer A, Gotoh H (2012) A 3D higher order Laplacian model for enhancement and stabilization of pressure calculation in 3D MPSbased simulations. Appl Ocean Res 37:120-126. https://doi.org/10. 1016/j.apor.2012.05.003

Khayyer A, Gotoh H, Shao SD (2008) Corrected incompressible SPH method for accurate water-surface tracking in breaking waves. Coast Eng 55(3):236-250. https://doi.org/10.1016/j.coastaleng.2007.10.001

Koshizuka S, Oka Y (1996) Moving-particle semi-implicit method for fragmentation of incompressible fluid. Nucl Sci Eng 123(3):421434. https://doi.org/10.13182/NSE96-A24205

Lee ES, Moulinec C, Xu R, Violeau D, Laurence D, Stansby PK (2008) Comparisons of weakly compressible and truly incompressible algorithms for the SPH mesh free particle method. J Comput Phys 227(18):8417-8436. https://doi.org/10.1016/j.jcp.2008.06.005

Lind SJ, Stansby PK (2016) High-order Eulerian incompressible smoothed particle hydrodynamics with transition to Lagrangian free surface motion. J Comput Phys 326:290-311. https://doi.org/10. 1016/j.jcp.2016.08.047

Lind SJ, Xu R, Stansby PK, Rogers BD (2012) Incompressible smoothed particle hydrodynamics for free-surface flows: a generalised diffusion-based algorithm for stability and validations for impulsive flows and propagating waves. J Comput Phys 231(4):1499-1523. https://doi.org/10.1016/j.jcp.2011.10.027

Lo EY, Shao S (2002) Simulation of near-shore solitary wave mechanics by an incompressible SPH method. Appl Ocean Res 24(5):275-286. https://doi.org/10.1016/S0141-1187(03)00002-6

Ma QW (2005a) MLPG method based on Rankine source solution for simulating nonlinear water waves. Comput Model Eng Sci 9(2): 193-210. https://doi.org/10.3970/cmes.2005.009.193

Ma QW (2005b) Meshless local Petrov-Galerkin method for twodimensional nonlinear water wave problems. J Comput Phys 205(2):611-625. https://doi.org/10.1016/j.jcp.2004.11.010

Ma QW (2008) A new meshless interpolation scheme for MLPG_R method. Comput Model Eng Sci 23(2):75-90. https://doi.org/10. 3970/cmes.2008.023.075

Ma QW, Zhou Y, Yan S (2016) A review on approaches to solving Poisson's equation in projection-based meshless methods for modelling strongly nonlinear water waves. J Ocean Eng Marine Energy 2(3):279-299. https://doi.org/10.1007/s40722-016-0063-5

Monaghan JJ (1994) Simulating free surface flows with SPH. J Comput Phys 110(2):399-406. https://doi.org/10.1006/jcph.1994.1034

Oger G, Doring M, Alessandrini B, Ferrant P (2007) An improved SPH method: towards higher order convergence. J Comput Phys 225(2): 1472-1492. https://doi.org/10.1016/j.jcp.2007.01.039

Quinlan NJ, Basa M, Lastiwka M (2006) Truncation error in mesh-free particle methods. Int J Numer Methods Eng 66(13):2064-2085. https://doi.org/10.1002/nme.1617

Schwaiger HF (2008) An implicit corrected SPH formulation for thermal diffusion with linear free surface boundary conditions. Int J Numer Methods Eng 75(6):647-671. https://doi.org/10.1002/nme.2266

Shao S, Ji C, Graham DI, Reeve DE, James PW, Chadwick AJ (2006) Simulation of wave overtopping by an incompressible SPH model. Coast Eng 53(9):723-735. https://doi.org/10.1016/j.coastaleng. 2006.02.005

Shao S, Lo EYM (2003) Incompressible SPH method for simulating Newtonian and non-Newtonian flows with free surface. Adv Water Resour 26(7):787-800. https://doi.org/10.1016/S03091708(03)00030-7

Tamai T, Koshizuka S (2014) Least squares moving particle semiimplicit method. Comput Part Mech 1(3):277-305. https://doi.org/ 10.1007/s40571-014-0029-0 
Tamai T, Murotani K, Koshizuka S (2017) On the consistency and convergence of particle-based meshfree discretization schemes for the Laplace operator. Comput Fluids 142:79-85. https://doi.org/10. 1016/j.compfluid.2016.02.012

Xu R, Stansby P, Laurence D (2009) Accuracy and stability in incompressible SPH (ISPH) based on the projection method and a new approach. J Comput Phys 228(18):6703-6025. https://doi.org/10. 1016/j.jcp.2009.05.032

Zheng X, Ma QW, Duan WY (2014) Incompressible SPH method based on Rankine source solution for violent water wave simulation. J
Comput Phys 276:291-314. https://doi.org/10.1016/j.jcp.2014.07. 036

Zheng X, Ma QW, Shao S (2018) Study on SPH Viscosity Term Formulations. Appl Sci 8(2):249. https://doi.org/10.3390/ app8020249

Zhou JT, Ma QW (2010) MLPG method based on Rankine source solution for modelling 3D breaking waves. Comput Model Eng Sci 56(2):179. https://doi.org/10.3970/cmes.2010.056.179 